\title{
Factors Affecting the Reinstatement of the Japanese Dental Hygienist: A Japanese Dental Hygienist Survey Conducted in 2019
}

\author{
Ayako Okada ${ }^{1,2}$, Yoshiaki Nomura ${ }^{1, *(D)}$, Yuki Ohara ${ }^{3,4}$ (D), Yuko Yamamoto ${ }^{5}$, Noriyasu Hosoya ${ }^{5}$, \\ Nobuhiro Hanada ${ }^{1}\left(\mathbb{D}\right.$ and Noriko Takei ${ }^{3}$
}

\section{check for}

updates

Citation: Okada, A.; Nomura, Y.; Ohara, Y.; Yamamoto, Y.; Hosoya, N.; Hanada, N.; Takei, N. Factors Affecting the Reinstatement of the Japanese Dental Hygienist: A Japanese Dental Hygienist Survey Conducted in 2019. Int. J. Environ Res. Public Health 2021, 18, 2049. https://doi.org/10.3390/ ijerph18042049

Academic Editor: Eduardo Bernabe

Received: 25 December 2020

Accepted: 17 February 2021

Published: 19 February 2021

Publisher's Note: MDPI stays neutra with regard to jurisdictional claims in published maps and institutional affiliations.

Copyright: (c) 2021 by the authors. Licensee MDPI, Basel, Switzerland. This article is an open access article distributed under the terms and conditions of the Creative Commons Attribution (CC BY) license (https:// creativecommons.org/licenses/by/ $4.0 /)$.
1 Department of Translational Research, Tsurumi University School of Dental Medicine, Yokohama 230-5801, Japan; okada-a@tsurumi-u.ac.jp (A.O.); hanada-n@tsurumi-u.ac.jp (N.H.)

2 Department of Operative Dentistry, Tsurumi University School of Dental Medicine, Yokohama 230-5801, Japan

3 Japan Dental Hygienists' Association, Tokyo 169-0071, Japan; yohara@tmig.or.jp (Y.O.); nori@pm-ms.tepm.jp (N.T.)

4 Tokyo Metropolitan Institute of Gerontology, Tokyo 173-0015, Japan

5 Department of Endodontology, Tsurumi University School of Dental Medicine, Yokohama 230-5801, Japan; yamamoto-y@tsurumi-u.ac.jp (Y.Y.); hosoya-n@tsurumi-u.ac.jp (N.H.)

* Correspondence: nomura-y@tsurumi-u.ac.jp; Tel.: +81-45-580-8462

\begin{abstract}
There is a shortage of dental hygienists for dental clinics in Japan. An ideal solution would be for dormant dental hygienists to re-enter the workforce. In this study, we identified the obstacles preventing these dental hygienists from re-entering the workforce. The Japan Dental Hygienists' Association surveyed all 16,113 members about their working conditions. Among the 101 items in the questionnaire, 11 items specifically targeted the reasons why dental hygienists leave their jobs. Among 8780 responses (54.5\%), 1063 were from members who had resigned from jobs as dental hygienists. Three hundred and fifty-five (34.4\%) answered "Yes" to the question "I would like to return to work if conditions are satisfactory." The most frequent cause for leaving a job was" Childbirth and child-rearing" (16.9\%). "Working status/working hours" (15.7\%) was the main obstacle to re-entry. Furthermore, dental hygienists with intentions to re-enter decided to leave their jobs most frequently because of "Childbirth and child-rearing" and "Working status/working hours." Improvement of the social support system for dental hygienists is required to ensure a sufficient supply of dental health services.
\end{abstract}

Keywords: dental hygienist; reinstatement; working environment

\section{Introduction}

Japan is a super-aging society with the proportion of people aged over 65 years being $28.4 \%$, while the working-age population (15-64 years old) accounts for $59.5 \%$ [1]. The proportion of working-age individuals is predicted to decrease even further with retirement and the low birthrate. To address this issue, the Japanese government is introducing reforms to the working environment. Recovering the decreasing birthrate and improving women's working environment are the main pillars of this reform. Child-rearing support systems have not been sufficient in Japan. The number of childcare facilities and kindergartens is far from sufficient [2]. Moreover, gender and income inequality remain [3].

Japanese law declares that the main task of dental hygienists is the improvement of oral health nationwide. The scope of the work of dental hygienists is oral care and health guidance under the supervision of a dentist, cleaning teeth, the mechanical removal of deposits on the tooth surface over the gingival margin, and assistance with dental treatment. Their dental treatment duties have expanded. There is a strong and increasing demand for oral healthcare in Japan, particularly for older adults. Pneumonia is one of 
the most common causes of death in Japan, and the crude death rate has been increasing. According to a demographics survey conducted in Japan, aspiration pneumonia resulted in 40,385 deaths in 2019, the sixth most common cause of death [4]. Several studies have shown that the maintenance of oral health and function has noticeable preventive effects against pneumonia, aspiration pneumonia [5-8], and influenza [9]. Previously, home-based dental care by dental hygienists alone was not allowed. Dentists had to accompany dental hygienists conducting dental care for older adults or handicapped person who could not attend dental clinics. The law of dental hygienists was amended to meet the demands of Japan's super-aging society. Dental hygienists can perform home-based dental care without a dentist once per week under the Japanese medical insurance and long-term care insurance system.

In addition, perioperative dental care has been introduced to the Japanese medical insurance system. Perioperative oral care management is an effective way to reduce disease complications and hospitalization costs. Many hospitals do not employ dental hygienists. Dentists or dental hygienists from private dental clinics visit hospitals for the oral care management of hospitalized patients. However, this scheme has not yet been thoroughly addressed.

The role of dental hygienists is vital for the prevention of these diseases. However, the number of dental hygienists per head of population is about 1 in 1000. The dental hygienist workforce is not sufficient to provide dental health services under the Japanese national medical insurance system. According to the national survey of Japan, 132,629 dental hygienists out of approximately 200,000 subjects who had licenses worked in 2018 [10]. The remaining $40 \%$ were not working in their field of expertise.

In Japan, dental services are sustained mainly by private dental clinics. As of 2018, there were 68,500 dental clinics in Japan [11]. A total of 120,068 (90.5\%) dental hygienists included part-time employees working in dental clinics [10]. The average number of dental hygienists per clinic was 1.6. There are turning points in a women's life-marriage, childbirth, and child-rearing - and the majority of dental hygienists in Japan are female. Working benefit systems are not usually utilized for dental hygienists, because private clinics do not have good benefit and welfare systems. This leads dental hygienists to have a career break. Promoting the re-employment of those who have left their dental hygienist career will increase the number of providers of dental care services.

A survey of the working conditions of dental hygienists is conducted by the Japan Dental Hygienists' Association every five years. This survey includes items to investigate why dental hygienists leave the professional field. This study aims to identify the obstacles faced by dental hygienists in re-entering their profession and propose countermeasures for supporting re-entry by statistical modeling.

\section{Materials and Methods}

\subsection{Survey Method}

The Japan Dental Hygienists' Association has been surveying the Employment Status of Japanese dental hygienists every 5 years since 1981. Questionnaires were distributed to all members of the Japan Dental Hygienists' Association on 16 October 2019 by postagepaid mail with a return envelope. The questionnaires received before the 11 November were used for the analysis. The questionnaire analyzed in this study is provided as supplemental material.

\subsection{Questionnaire}

The questionnaire consisted of 101 items concerning demographic factors, employment situation, job description, and willingness to work.

There were ten specific items targeted at dental hygienists who were leaving their jobs as dental hygienists. In this study, we analyzed these ten specific items.

These items are as follows:

(1) Why did you decide to leave the workplace where you were last working as a dental hygienist? Select all that apply and mark the best answer separately. 
1. Dissatisfaction with work content, 2. Payment/welfare, 3. Relationship with the manager, 4. Relationships with seniors, 5 . Relationships with colleagues, 6 . Working status/working hours, 7. Long working hours/overwork, 8. Family matters, 9. Job transfer of family member, 10. Marriage, 11 . Childbirth/child-rearing, 12 . Nursing care for family members, 13. Personal health problem, 14. To improve the level of work content, 15. Inadequate training program, 16 . Limits of personal skills, 17 . The job was not rewarding, 18. Interest in the different fields, 19. Others.

(2) What do you think should have been improved about your workplace where you were working last? Select all that apply and mark the best answer separately.

1. Improvement of working conditions (base up, regular pay raise, etc.), 2. Reduction of workload, 3. Interpersonal relationships at the workplace, 4 . Reduction of working hours, 5 . Taking a vacation, 6 . Enhancement of child-rearing support, 7. Enhancement of nursing care support, 8. Evaluation of expertise, qualifications, etc., 9. Enriching opportunities to improve personal skills, through education, training, etc., 10. Introduction of various work styles and working hours, 11. Enhancement of the medical safety system, 12. Ensuring employment stability, 13. Enrichment of employee benefits, 14. Nothing in particular, 15. Other.

(3) How long has it been since you left your job as a dental hygienist?

(4) Do you want to start working as a dental hygienist again? Select one.

1. I want to re-enter as soon as possible, 2. I want to re-enter if conditions are satisfied, 3. I do not intend to, 4 . I do not know.

(5) Do you want to start working again at the same workplace where you were last working as a dental hygienist? Select one.

1. I want to re-enter as soon as possible, 2. I want to re-enter if conditions are satisfied, 3. I do not intend to 4 . I do not know.

(6) If you were to start working again, how would you find a workplace? Multiple choice.

1. Labor agency, 2. Job magazines, 3. Recommendation from a friend/acquaintance, 4. Recommendation from a dental hygienist school, 5 . Internet, 6 . Free referral by the Dental Association and Dental Hygienist Association, 7. Human resource dispatch companies, 8. Other.

(7) (1) Are there any obstacles that are making it difficult for you to start working again as a dental hygienist?

1. Yes, 2. No.

(2) What are these obstacles? Multiple choice.

1. Salary, 2. Working hours, 3. My skills, 4. No consultation, 5. Aging, 6. Others.

(8) Where do you want to start working again as a dental hygienist? Multiple choice.

1. Clinic, 2. Hospital/university hospital, 3. Clinic for special needs dentistry, 4. Administration, 5. Dental hygienist education and training institution, 6. Clinic attached to the company, 7. Companies/business establishments, 8. Dental health examination/health activity organization, 9 . Social welfare facility, 10. Long-term care insurance facilities, etc. (Please mark the relevant facilities with a circle), 11. Regional comprehensive support center, etc., 12. Research institute, 13. Other.

(9) What are the areas of your interest? Multiple choice.

1. Community health, 2. Home-based dental care (management guidance for inhome care, home-visit oral hygiene management, etc.), 3. Geriatric dentistry, 4. Special needs dentistry, 5. Occupational dental health, 6 . Dental hygienist education and training institution, 7. Nothing in particular, 8. Other.

(10) Would you want to participate in a lecture-based course for re-entry?

1. Yes, 2. No. 
(11) What kind of lecture-based courses would you want to attend? Multiple choice and choose the best answer.

1. Professional oral care techniques, 2. Scaling and route planning, 3. Dental caries prevention and control, 4 . Dental health guidance for individuals, 5 . Dental health guidance for group, 6. Handling of dental materials, chairside assistant, 7. Chairside assistant techniques, such as snap impression, polishing of filling materials, rubber dam, etc., 8 . Counseling skills, 9. Dysphagia rehabilitation, 10. Myofunctional therapy, 11. Nursing care skills, 12. Other.

The questionnaire is available in Japanese from the Japan Dental Hygienists' Association [10].

\subsection{Statistical Analysis}

Descriptive statistics and cross-tabulations with age groups were summarized for the questions presented above. Chi-square tests were used to find out the statistical significance. A logit model of log-linear analysis was used to identify statistically significant cells. To visualize the relations, a correspondence analysis was conducted. The results are expressed as biplots. SPSS Statistics Ver 24.0 (IBM, Tokyo, Japan) was used for the analysis.

\subsection{Ethics Approval and Consent to Participate}

Informed written consent was obtained from all participants. This study was approved by the Ethics Committee of the Tsurumi University School of Dental Medicine (approval number: 1837) and conducted in accordance with the Declaration of Helsinki.

\section{Results}

The Japan Dental Hygienists' Association distributed the questionnaires to 16,113 of its members, and 8780 responded (collection rate: 54.5\%). Among them, there were 1063 dormant dental hygienists. In this study, the data analysis included 1063 dental hygienists. Their mean age was $52.36 \pm 13.06$ years. They were all women.

Table 1 shows a cross-tabulation of the specific questionnaire answered by the dental hygienists who were leaving their jobs. They were classified into age groups of $\leq 29,30-39$, $40-49,50-59$, and $60-70$ years. Among 851 dental hygienists, 351 (34.4\%) answered that "They would like to return to work if conditions were satisfied." Approximately $40 \%$ of those in the 30-39 year age group were willing to re-enter the workforce (as soon as possible and if conditions are satisfied).

Table 1. Cross-tabulation of the items investigated in this study against age groups.

\begin{tabular}{|c|c|c|c|c|c|c|c|c|}
\hline Age & $20 \mathrm{~s}$ & $30 \mathrm{~s}$ & $40 \mathrm{~s}$ & $50 \mathrm{~s}$ & $60 \mathrm{~s}$ & $70 \mathrm{~s}$ & Total & $p$-Value \\
\hline \multicolumn{9}{|c|}{ Do you want to start working as a dental hygienist again? } \\
\hline $\begin{array}{l}\text { I want to re-enter as } \\
\text { soon as possible }\end{array}$ & $\begin{array}{c}9 \\
(21.4 \%)\end{array}$ & $\begin{array}{c}12 \\
(7.0 \%)\end{array}$ & $\begin{array}{c}7 \\
(3.7 \%)\end{array}$ & $\begin{array}{c}9 \\
(3.2 \%)\end{array}$ & $\begin{array}{c}3 \\
(1.1 \%)\end{array}$ & $\begin{array}{c}0 \\
(0.0 \%)\end{array}$ & $\begin{array}{c}40 \\
(3.9 \%)\end{array}$ & \multirow{4}{*}{$<0.001$} \\
\hline $\begin{array}{l}\text { I want to re-enter if conditions } \\
\text { are satisfied }\end{array}$ & $\begin{array}{c}26 \\
(61.9 \%)\end{array}$ & $\begin{array}{c}107 \\
(62.6 \%)\end{array}$ & $\begin{array}{c}90 \\
(48.1 \%)\end{array}$ & $\begin{array}{c}90 \\
(32.3 \%)\end{array}$ & $\begin{array}{c}36 \\
(13.2 \%)\end{array}$ & $\begin{array}{c}2 \\
(2.9 \%)\end{array}$ & $\begin{array}{c}351 \\
(34.4 \%)\end{array}$ & \\
\hline I do not intend to & $\begin{array}{c}2 \\
(4.8 \%)\end{array}$ & $\begin{array}{c}22 \\
(12.9 \%)\end{array}$ & $\begin{array}{c}49 \\
(26.2 \%)\end{array}$ & $\begin{array}{c}111 \\
(39.8 \%)\end{array}$ & $\begin{array}{c}195 \\
(71.4 \%)\end{array}$ & $\begin{array}{c}65 \\
(94.2 \%)\end{array}$ & $\begin{array}{c}444 \\
(43.5 \%)\end{array}$ & \\
\hline I do not know & $\begin{array}{c}5 \\
(11.9 \%)\end{array}$ & $\begin{array}{c}30 \\
(17.5 \%)\end{array}$ & $\begin{array}{c}41 \\
(21.9 \%)\end{array}$ & $\begin{array}{c}69 \\
(24.7 \%)\end{array}$ & $\begin{array}{c}39 \\
(14.3 \%)\end{array}$ & $\begin{array}{c}2 \\
(2.9 \%)\end{array}$ & $\begin{array}{c}186 \\
(18.2 \%)\end{array}$ & \\
\hline
\end{tabular}


Table 1. Cont.

\begin{tabular}{|c|c|c|c|c|c|c|c|c|}
\hline Age & $20 \mathrm{~s}$ & $30 \mathrm{~s}$ & $40 \mathrm{~s}$ & $50 \mathrm{~s}$ & $60 \mathrm{~s}$ & $70 \mathrm{~s}$ & Total & $p$-Value \\
\hline \multicolumn{9}{|c|}{ Why did you decide to leave the workplace where you were last working as a dental hygienist? } \\
\hline $\begin{array}{l}\text { Dissatisfaction with } \\
\text { work content }\end{array}$ & $\begin{array}{c}6 \\
(14.0 \%)\end{array}$ & $\begin{array}{c}27 \\
(15.4 \%)\end{array}$ & $\begin{array}{c}25 \\
(13.2 \%)\end{array}$ & $\begin{array}{c}23 \\
(8.0 \%)\end{array}$ & $\begin{array}{c}13 \\
(4.6 \%)\end{array}$ & $\begin{array}{c}4 \\
(5.6 \%)\end{array}$ & $\begin{array}{c}98 \\
(9.4 \%)\end{array}$ & 0.001 \\
\hline Payment/welfare & $\begin{array}{c}6 \\
(14 \%) \\
\end{array}$ & $\begin{array}{c}28 \\
(16.0 \%)\end{array}$ & $\begin{array}{c}33 \\
(17.5 \%)\end{array}$ & $\begin{array}{c}47 \\
(16.4 \%)\end{array}$ & $\begin{array}{c}20 \\
(7.1 \%)\end{array}$ & $\begin{array}{c}1 \\
(1.4 \%)\end{array}$ & $\begin{array}{c}135 \\
(12.9 \%)\end{array}$ & $<0.001$ \\
\hline Relationship with the manager & $\begin{array}{c}15 \\
(34.9 \%)\end{array}$ & $\begin{array}{c}34 \\
(19.4 \%)\end{array}$ & $\begin{array}{c}42 \\
(22.2 \%)\end{array}$ & $\begin{array}{c}45 \\
(15.7 \%)\end{array}$ & $\begin{array}{c}27 \\
(9.6 \%)\end{array}$ & $\begin{array}{c}1 \\
(1.4 \%)\end{array}$ & $\begin{array}{c}164 \\
(15.7 \%)\end{array}$ & $<0.001$ \\
\hline Relationships with seniors & $\begin{array}{c}9 \\
(20.9 \%)\end{array}$ & $\begin{array}{c}21 \\
(12.0 \%)\end{array}$ & $\begin{array}{c}23 \\
(12.2 \%)\end{array}$ & $\begin{array}{c}10 \\
(3.5 \%)\end{array}$ & $\begin{array}{c}2 \\
(0.7 \%)\end{array}$ & $\begin{array}{c}0 \\
(0.0 \%)\end{array}$ & $\begin{array}{c}65 \\
(6.2 \%)\end{array}$ & $<0.001$ \\
\hline Relationships with colleagues & $\begin{array}{c}3 \\
(7.0 \%)\end{array}$ & $\begin{array}{c}13 \\
(7.4 \%)\end{array}$ & $\begin{array}{c}23 \\
(12.2 \%)\end{array}$ & $\begin{array}{c}26 \\
(9.1 \%)\end{array}$ & $\begin{array}{c}15 \\
(5.4 \%)\end{array}$ & $\begin{array}{c}1 \\
(1.4 \%)\end{array}$ & $\begin{array}{c}81 \\
(7.8 \%)\end{array}$ & 0.031 \\
\hline Working status/working hours & $\begin{array}{c}8 \\
(18.6 \%)\end{array}$ & $\begin{array}{c}24 \\
(13.7 \%)\end{array}$ & $\begin{array}{c}39 \\
(20.6 \%)\end{array}$ & $\begin{array}{c}33 \\
(11.5 \%)\end{array}$ & $\begin{array}{c}19 \\
(6.8 \%)\end{array}$ & $\begin{array}{c}1 \\
(1.4 \%)\end{array}$ & $\begin{array}{c}124 \\
(11.9 \%)\end{array}$ & $<0.001$ \\
\hline Long working hours/overwork & $\begin{array}{c}5 \\
(11.6 \%)\end{array}$ & $\begin{array}{c}21 \\
(12.0 \%)\end{array}$ & $\begin{array}{c}21 \\
(11.1 \%)\end{array}$ & $\begin{array}{c}15 \\
(5.2 \%)\end{array}$ & $\begin{array}{c}10 \\
(3.6 \%)\end{array}$ & $\begin{array}{c}2 \\
(2.8 \%)\end{array}$ & $\begin{array}{c}74 \\
(7.1 \%)\end{array}$ & 0.001 \\
\hline Family matters & $\begin{array}{c}1 \\
(2.3 \%)\end{array}$ & $\begin{array}{c}17 \\
(9.7 \%)\end{array}$ & $\begin{array}{c}24 \\
(12.7 \%)\end{array}$ & $\begin{array}{c}43 \\
(15.0 \%)\end{array}$ & $\begin{array}{c}39 \\
(13.9 \%)\end{array}$ & $\begin{array}{c}13 \\
(18.1 \%)\end{array}$ & $\begin{array}{c}137 \\
(13.1 \%)\end{array}$ & 0.116 \\
\hline Job transfer of family member & $\begin{array}{c}2 \\
(4.7 \%)\end{array}$ & $\begin{array}{c}12 \\
(6.9 \%)\end{array}$ & $\begin{array}{c}0 \\
(0.0 \%)\end{array}$ & $\begin{array}{c}6 \\
(2.1 \%)\end{array}$ & $\begin{array}{c}3 \\
(1.1 \%)\end{array}$ & $\begin{array}{c}3 \\
(4.2 \%)\end{array}$ & $\begin{array}{c}26 \\
(2.5 \%)\end{array}$ & $<0.001$ \\
\hline Marriage & $\begin{array}{c}2 \\
(4.7 \%)\end{array}$ & $\begin{array}{c}27 \\
(15.4 \%)\end{array}$ & $\begin{array}{c}30 \\
(15.9 \%) *\end{array}$ & $\begin{array}{c}36 \\
(12.6 \%)\end{array}$ & $\begin{array}{c}22 \\
(7.9 \%)\end{array}$ & $\begin{array}{c}4 \\
(5.6 \%)\end{array}$ & $\begin{array}{c}121 \\
(11.6 \%)\end{array}$ & 0.012 \\
\hline Childbirth/child-rearing & $\begin{array}{c}15 \\
(34.9 \%) *\end{array}$ & $\begin{array}{c}71 \\
(40.6 \%)\end{array}$ & $\begin{array}{c}42 \\
(22.2 \%) *\end{array}$ & $\begin{array}{c}33 \\
(11.5 \%)\end{array}$ & $\begin{array}{c}12 \\
(4.3 \%)\end{array}$ & $\begin{array}{c}4 \\
(5.6 \%)\end{array}$ & $\begin{array}{c}177 \\
(16.9 \%)\end{array}$ & $<0.001$ \\
\hline Nursing care for family member & $\begin{array}{c}1 \\
(2.3 \%)\end{array}$ & $\begin{array}{c}3 \\
(1.7 \%) *\end{array}$ & $\begin{array}{c}7 \\
(3.7 \%)\end{array}$ & $\begin{array}{c}26 \\
(9.1 \%)\end{array}$ & $\begin{array}{c}34 \\
(12.1 \%)\end{array}$ & $\begin{array}{c}7 \\
(9.7 \%)\end{array}$ & $\begin{array}{c}78 \\
(7.5 \%)\end{array}$ & $<0.001$ \\
\hline Personal health & $\begin{array}{c}9 \\
(20.9 \%)\end{array}$ & $\begin{array}{c}24 \\
(13.7 \%)\end{array}$ & $\begin{array}{c}30 \\
(15.9 \%)\end{array}$ & $\begin{array}{c}31 \\
(10.8 \%)\end{array}$ & $\begin{array}{c}46 \\
(16.4 \%)\end{array}$ & $\begin{array}{c}11 \\
(15.3 \%)\end{array}$ & $\begin{array}{c}151 \\
(14.4 \%)\end{array}$ & 0.327 \\
\hline $\begin{array}{l}\text { To improve the level } \\
\text { of work content }\end{array}$ & $\begin{array}{c}6 \\
(14.0 \%) *\end{array}$ & $\begin{array}{c}8 \\
(4.6 \%)\end{array}$ & $\begin{array}{c}7 \\
(3.7 \%)\end{array}$ & $\begin{array}{c}5 \\
(1.7 \%)\end{array}$ & $\begin{array}{c}3 \\
(1.1 \%)\end{array}$ & $\begin{array}{c}0 \\
(0.0 \%)\end{array}$ & $\begin{array}{c}29 \\
(2.8 \%)\end{array}$ & $<0.001$ \\
\hline Inadequate training program & $\begin{array}{c}4 \\
(9.3 \%)\end{array}$ & $\begin{array}{c}8 \\
(4.6 \%)\end{array}$ & $\begin{array}{c}8 \\
(4.2 \%)\end{array}$ & $\begin{array}{c}6 \\
(2.1 \%)\end{array}$ & $\begin{array}{c}3 \\
(1.1 \%)\end{array}$ & $\begin{array}{c}0 \\
(0.0 \%)\end{array}$ & $\begin{array}{c}29 \\
(2.8 \%)\end{array}$ & 0.007 \\
\hline Limits of personal skills & $\begin{array}{c}4 \\
(9.3 \%) \\
\end{array}$ & $\begin{array}{c}14 \\
(8.0 \%) \\
\end{array}$ & $\begin{array}{c}14 \\
(7.4 \%) \\
\end{array}$ & $\begin{array}{c}16 \\
(5.6 \%) \\
\end{array}$ & $\begin{array}{c}10 \\
(3.6 \%) \\
\end{array}$ & $\begin{array}{c}5 \\
(6.9 \%) \\
\end{array}$ & $\begin{array}{c}63 \\
(6.0 \%) \\
\end{array}$ & 0.323 \\
\hline The job was not rewarding & $\begin{array}{c}3 \\
(7.0 \%) \\
\end{array}$ & $\begin{array}{c}14 \\
(8.0 \%) \\
\end{array}$ & $\begin{array}{c}14 \\
(7.4 \%)\end{array}$ & $\begin{array}{c}13 \\
(4.5 \%)\end{array}$ & $\begin{array}{c}6 \\
(2.1 \%)\end{array}$ & $\begin{array}{c}1 \\
(1.4 \%)\end{array}$ & $\begin{array}{c}51 \\
(4.9 \%)\end{array}$ & 0.022 \\
\hline Interest in a different field & $\begin{array}{c}1 \\
(2.3 \%)\end{array}$ & $\begin{array}{c}17 \\
(9.7 \%)\end{array}$ & $\begin{array}{c}19 \\
(10.1 \%)\end{array}$ & $\begin{array}{c}52 \\
(18.2 \%) *\end{array}$ & $\begin{array}{c}21 \\
(7.5 \%)\end{array}$ & $\begin{array}{c}4 \\
(5.6 \%)\end{array}$ & $\begin{array}{c}114 \\
(10.9 \%)\end{array}$ & $<0.001$ \\
\hline Others & $\begin{array}{c}7 \\
(16.3 \%) *\end{array}$ & $\begin{array}{c}31 \\
(17.7 \%) *\end{array}$ & $\begin{array}{c}44 \\
(23.3 \%)\end{array}$ & $\begin{array}{c}74 \\
(25.9 \%)\end{array}$ & $\begin{array}{c}123 \\
(43.9 \%)\end{array}$ & $\begin{array}{c}35 \\
(48.6 \%)\end{array}$ & $\begin{array}{c}314 \\
(30.0 \%)\end{array}$ & $<0.001$ \\
\hline
\end{tabular}


Table 1. Cont.

\begin{tabular}{|c|c|c|c|c|c|c|c|c|}
\hline Age & $20 \mathrm{~s}$ & $30 \mathrm{~s}$ & $40 \mathrm{~s}$ & $50 \mathrm{~s}$ & $60 \mathrm{~s}$ & $70 \mathrm{~s}$ & Total & $p$-Value \\
\hline \multicolumn{9}{|c|}{ What do you think should have been improved about your workplace where you were working last? } \\
\hline $\begin{array}{l}\text { Improvement of working } \\
\text { conditions (base up, regular } \\
\text { pay raise, etc.,) }\end{array}$ & $\begin{array}{c}9 \\
(21.4 \%) *\end{array}$ & $\begin{array}{c}56 \\
(32.7 \%) *\end{array}$ & $\begin{array}{c}48 \\
(26.1 \%) *\end{array}$ & $\begin{array}{c}79 \\
(28.2 \%) *\end{array}$ & $\begin{array}{c}62 \\
(23.0 \%) *\end{array}$ & $\begin{array}{c}4 \\
(6.1 \%)\end{array}$ & $\begin{array}{c}258 \\
(25.5 \%)\end{array}$ & 0.001 \\
\hline Reduction of work load & $\begin{array}{c}10 \\
(23.8 \%)\end{array}$ & $\begin{array}{c}36 \\
(21.1 \%) \\
\end{array}$ & $\begin{array}{c}29 \\
(15.8 \%) \\
\end{array}$ & $\begin{array}{c}29 \\
(10.4 \%)\end{array}$ & $\begin{array}{c}36 \\
(13.3 \%)\end{array}$ & $\begin{array}{c}7 \\
(10.6 \%)\end{array}$ & $\begin{array}{c}147 \\
(14.5 \%)\end{array}$ & 0.015 \\
\hline $\begin{array}{l}\text { Interpersonal relationships at } \\
\text { the workplace }\end{array}$ & $\begin{array}{c}22 \\
(52.4 \%) *\end{array}$ & $\begin{array}{c}61 \\
(35.7 \%) *\end{array}$ & $\begin{array}{c}74 \\
(40.2 \%) *\end{array}$ & $\begin{array}{c}79 \\
(28.2 \%) *\end{array}$ & $\begin{array}{c}48 \\
(17.8 \%) *\end{array}$ & $\begin{array}{c}1 \\
(1.5 \%)\end{array}$ & $\begin{array}{c}285 \\
(28.1 \%)\end{array}$ & $<0.001$ \\
\hline Reduction of working hours & $\begin{array}{c}10 \\
(23.8 \%) *\end{array}$ & $\begin{array}{c}40 \\
(23.4 \%) *\end{array}$ & $\begin{array}{c}31 \\
(16.8 \%)\end{array}$ & $\begin{array}{c}36 \\
(12.9 \%)\end{array}$ & $\begin{array}{c}22 \\
(8.1 \%)\end{array}$ & $\begin{array}{c}6 \\
(9.1 \%)\end{array}$ & $\begin{array}{c}145 \\
(14.3 \%)\end{array}$ & $<0.001$ \\
\hline Taking a vacation & $\begin{array}{c}10 \\
(23.8 \%)^{*}\end{array}$ & $\begin{array}{c}43 \\
(25.1 \%)^{*}\end{array}$ & $\begin{array}{c}37 \\
(20.1 \%) \\
\end{array}$ & $\begin{array}{c}57 \\
(20.4 \%) * \\
\end{array}$ & $\begin{array}{c}28 \\
(10.4 \%) \\
\end{array}$ & $\begin{array}{c}6 \\
(9.1 \%) \\
\end{array}$ & $\begin{array}{c}181 \\
(17.9 \%) \\
\end{array}$ & $<0.001$ \\
\hline $\begin{array}{l}\text { Enhancement of } \\
\text { child-rearing support }\end{array}$ & $\begin{array}{c}6 \\
(14.3 \%) \\
\end{array}$ & $\begin{array}{c}33 \\
(19.3 \%) *\end{array}$ & $\begin{array}{c}15 \\
(8.2 \%) \\
\end{array}$ & $\begin{array}{c}11 \\
(3.9 \%) \\
\end{array}$ & $\begin{array}{c}7 \\
(2.6 \%) \\
\end{array}$ & $\begin{array}{c}3 \\
(4.5 \%) \\
\end{array}$ & $\begin{array}{c}75 \\
(7.4 \%) \\
\end{array}$ & $<0.001$ \\
\hline $\begin{array}{l}\text { Enhancement of nursing } \\
\text { care support }\end{array}$ & $\begin{array}{c}0 \\
(0.0 \%)\end{array}$ & $\begin{array}{c}0 \\
(0.0 \%) *\end{array}$ & $\begin{array}{c}3 \\
(1.6 \%)\end{array}$ & $\begin{array}{c}8 \\
(2.9 \%)\end{array}$ & $\begin{array}{c}11 \\
(4.1 \%)\end{array}$ & $\begin{array}{c}4 \\
(6.1 \%)\end{array}$ & $\begin{array}{c}26 \\
(2.6 \%)\end{array}$ & 0.034 \\
\hline $\begin{array}{l}\text { Evaluation of expertise, } \\
\text { qualifications, etc. }\end{array}$ & $\begin{array}{c}4 \\
(9.5 \%)\end{array}$ & $\begin{array}{c}29 \\
(17.0 \%)\end{array}$ & $\begin{array}{c}33 \\
(17.9 \%)\end{array}$ & $\begin{array}{c}41 \\
(14.6 \%)\end{array}$ & $\begin{array}{c}54 \\
(20.0 \%)\end{array}$ & $\begin{array}{c}13 \\
(19.7 \%)\end{array}$ & $\begin{array}{c}174 \\
(17.2 \%)\end{array}$ & 0.431 \\
\hline $\begin{array}{l}\text { Enriching opportunities to } \\
\text { improve personal skills through } \\
\text { education, training, etc. }\end{array}$ & $\begin{array}{c}11 \\
(26.2 \%) *\end{array}$ & $\begin{array}{c}29 \\
(17.0 \%)\end{array}$ & $\begin{array}{c}27 \\
(14.7 \%)\end{array}$ & $\begin{array}{c}36 \\
(12.9 \%)\end{array}$ & $\begin{array}{c}35 \\
(13.0 \%)\end{array}$ & $\begin{array}{c}6 \\
(9.1 \%)\end{array}$ & $\begin{array}{c}144 \\
(14.2 \%)\end{array}$ & 0.145 \\
\hline $\begin{array}{l}\text { Introduction of various work } \\
\text { styles and working hours }\end{array}$ & $\begin{array}{c}3 \\
(7.1 \%)\end{array}$ & $\begin{array}{c}25 \\
(14.6 \%) *\end{array}$ & $\begin{array}{c}17 \\
(9.2 \%)\end{array}$ & $\begin{array}{c}24 \\
(8.6 \%)\end{array}$ & $\begin{array}{c}14 \\
(5.2 \%)\end{array}$ & $\begin{array}{c}1 \\
(1.5 \%)\end{array}$ & $\begin{array}{c}84 \\
(8.3 \%)\end{array}$ & 0.005 \\
\hline $\begin{array}{l}\text { Enhancement of the medical } \\
\text { safety system }\end{array}$ & $\begin{array}{c}5 \\
(11.9 \%)\end{array}$ & $\begin{array}{c}20 \\
(11.7 \%)\end{array}$ & $\begin{array}{c}15 \\
(8.2 \%)\end{array}$ & $\begin{array}{c}24 \\
(8.6 \%)\end{array}$ & $\begin{array}{c}11 \\
(4.1 \%)\end{array}$ & $\begin{array}{c}2 \\
(3.0 \%)\end{array}$ & $\begin{array}{c}77 \\
(7.6 \%)\end{array}$ & 0.03 \\
\hline Ensuring employment stability & $\begin{array}{c}3 \\
(7.1 \%)\end{array}$ & $\begin{array}{c}19 \\
(11.1 \%)\end{array}$ & $\begin{array}{c}14 \\
(7.6 \%)\end{array}$ & $\begin{array}{c}25 \\
(8.9 \%)\end{array}$ & $\begin{array}{c}22 \\
(8.1 \%)\end{array}$ & $\begin{array}{c}2 \\
(3.0 \%)\end{array}$ & $\begin{array}{c}85 \\
(8.4 \%)\end{array}$ & 0.484 \\
\hline $\begin{array}{c}\text { Enrichment of } \\
\text { employee benefits }\end{array}$ & $\begin{array}{c}6 \\
(14.3 \%) \\
\end{array}$ & $\begin{array}{c}32 \\
(18.7 \%) \\
\end{array}$ & $\begin{array}{c}27 \\
(14.7 \%) \\
\end{array}$ & $\begin{array}{c}49 \\
(17.5 \%) \\
\end{array}$ & $\begin{array}{c}34 \\
(12.6 \%) \\
\end{array}$ & $\begin{array}{c}7 \\
(10.6 \%) \\
\end{array}$ & $\begin{array}{c}155 \\
(15.3 \%) \\
\end{array}$ & 0.378 \\
\hline Nothing in particular & $\begin{array}{c}5 \\
(11.9 \%) *\end{array}$ & $\begin{array}{c}26 \\
(15.2 \%) *\end{array}$ & $\begin{array}{c}34 \\
(18.5 \%) *\end{array}$ & $\begin{array}{c}67 \\
(23.9 \%) *\end{array}$ & $\begin{array}{c}86 \\
(31.9 \%) \\
\end{array}$ & $\begin{array}{c}29 \\
(43.9 \%)\end{array}$ & $\begin{array}{c}247 \\
(24.4 \%) \\
\end{array}$ & $<0.001$ \\
\hline Other & $\begin{array}{c}4 \\
(9.5 \%)\end{array}$ & $\begin{array}{c}15 \\
(8.8 \%)\end{array}$ & $\begin{array}{c}18 \\
(9.8 \%)\end{array}$ & $\begin{array}{c}17 \\
(6.1 \%)\end{array}$ & $\begin{array}{c}24 \\
(8.9 \%)\end{array}$ & $\begin{array}{c}4 \\
(6.1 \%)\end{array}$ & $\begin{array}{c}82 \\
(8.1 \%)\end{array}$ & 0.69 \\
\hline \multicolumn{9}{|c|}{ Are there any obstacles that are making it difficult for you to start working again as a dental hygienist? } \\
\hline Yes & $\begin{array}{c}24 \\
(72.7 \%)\end{array}$ & $\begin{array}{c}106 \\
(89.1 \%)\end{array}$ & $\begin{array}{c}84 \\
(87.5 \%)\end{array}$ & $\begin{array}{c}88 \\
(90.7 \%)\end{array}$ & $\begin{array}{c}34 \\
(87.2 \%)\end{array}$ & $\begin{array}{c}1 \\
(50.0 \%)\end{array}$ & $\begin{array}{c}337 \\
(87.3 \%)\end{array}$ & 0.07 \\
\hline
\end{tabular}


Table 1. Cont.

\begin{tabular}{|c|c|c|c|c|c|c|c|c|}
\hline Age & $20 \mathrm{~s}$ & $30 \mathrm{~s}$ & $40 \mathrm{~s}$ & $50 \mathrm{~s}$ & $60 \mathrm{~s}$ & $70 \mathrm{~s}$ & Total & $p$-Value \\
\hline \multicolumn{9}{|c|}{ What are these obstacles? } \\
\hline Salary & $\begin{array}{c}8 \\
(33.3 \%)\end{array}$ & $\begin{array}{c}32 \\
(30.5 \%)\end{array}$ & $\begin{array}{c}23 \\
(27.4 \%)\end{array}$ & $\begin{array}{c}18 \\
(20.9 \%)\end{array}$ & $\begin{array}{c}3 \\
(8.8 \%)\end{array}$ & $\begin{array}{c}0 \\
(0.0 \%)\end{array}$ & $\begin{array}{c}84 \\
(25.1 \%)\end{array}$ & 0.125 \\
\hline Working hours & $\begin{array}{c}16 \\
(66.7 \%)\end{array}$ & $\begin{array}{c}81 \\
(77.1 \%)\end{array}$ & $\begin{array}{c}59 \\
(70.2 \%)\end{array}$ & $\begin{array}{c}24 \\
(27.9 \%)\end{array}$ & $\begin{array}{c}13 \\
(38.2 \%)\end{array}$ & $\begin{array}{c}1 \\
(100 \%)\end{array}$ & $\begin{array}{c}194 \\
(58.1 \%)\end{array}$ & $<0.001$ \\
\hline My skills & $\begin{array}{c}18 \\
(75.0 \%)\end{array}$ & $\begin{array}{c}43 \\
(41.0 \%)\end{array}$ & $\begin{array}{c}41 \\
(48.8 \%)\end{array}$ & $\begin{array}{c}47 \\
(54.7 \%)\end{array}$ & $\begin{array}{c}11 \\
(32.4 \%)\end{array}$ & $\begin{array}{c}0 \\
(0.0 \%)\end{array}$ & $\begin{array}{c}160 \\
(47.9 \%)\end{array}$ & 0.011 \\
\hline No consultation & $\begin{array}{c}4 \\
(16.7 \%)\end{array}$ & $\begin{array}{c}5 \\
(4.8 \%)\end{array}$ & $\begin{array}{c}6 \\
(7.1 \%)\end{array}$ & $\begin{array}{c}9 \\
(10.5 \%)\end{array}$ & $\begin{array}{c}4 \\
(11.8 \%)\end{array}$ & $\begin{array}{c}0 \\
(0.0 \%)\end{array}$ & $\begin{array}{c}28 \\
(8.4 \%)\end{array}$ & 0.393 \\
\hline Aging & $\begin{array}{c}0 \\
(0.0 \%) *\end{array}$ & $\begin{array}{c}4 \\
(3.8 \%) *\end{array}$ & $\begin{array}{c}16 \\
(19.0 \%)\end{array}$ & $\begin{array}{c}51 \\
(59.3 \%)\end{array}$ & $\begin{array}{c}21 \\
(61.8 \%)\end{array}$ & $\begin{array}{c}1 \\
(100 \%)\end{array}$ & $\begin{array}{c}93 \\
(27.8 \%)\end{array}$ & $<0.001$ \\
\hline Others & $\begin{array}{c}4 \\
(16.7 \%)\end{array}$ & $\begin{array}{c}37 \\
(35.2 \%)\end{array}$ & $\begin{array}{c}25 \\
(29.8 \%)\end{array}$ & $\begin{array}{c}23 \\
(26.7 \%)\end{array}$ & $\begin{array}{c}7 \\
(20.6 \%)\end{array}$ & $\begin{array}{c}0 \\
(0.0 \%)\end{array}$ & $\begin{array}{c}96 \\
(28.7 \%)\end{array}$ & 0.348 \\
\hline \multicolumn{9}{|c|}{ Would you want to participate in a lecture-based course for re-entry? } \\
\hline Yes & $\begin{array}{c}27 \\
(62.8 \%)\end{array}$ & $\begin{array}{c}108 \\
(63.5 \%)\end{array}$ & $\begin{array}{c}116 \\
(61.1 \%)\end{array}$ & $\begin{array}{c}152 \\
(53.1 \%)\end{array}$ & $\begin{array}{c}85 \\
(31.3 \%)\end{array}$ & $\begin{array}{c}9 \\
(13.4 \%)\end{array}$ & $\begin{array}{c}497 \\
(48.3 \%)\end{array}$ & $<0.001$ \\
\hline \multicolumn{9}{|c|}{ What kind of lecture courses would you want to attend? } \\
\hline $\begin{array}{l}\text { Professional oral } \\
\text { care techniques }\end{array}$ & $\begin{array}{c}7 \\
(25.9 \%)\end{array}$ & $\begin{array}{c}62 \\
(57.4 \%) *\end{array}$ & $\begin{array}{c}66 \\
(56.9 \%) *\end{array}$ & $\begin{array}{c}63 \\
(41.4 \%)\end{array}$ & $\begin{array}{c}26 \\
(30.6 \%)\end{array}$ & $\begin{array}{c}1 \\
(11.1 \%)\end{array}$ & $\begin{array}{c}225 \\
(45.3 \%)\end{array}$ & $<0.001$ \\
\hline Scaling and route planning & $\begin{array}{c}21 \\
(77.8 \%) *\end{array}$ & $\begin{array}{c}72 \\
(66.7 \%) *\end{array}$ & $\begin{array}{c}62 \\
(53.4 \%) *\end{array}$ & $\begin{array}{c}40 \\
(26.3 \%)\end{array}$ & $\begin{array}{c}12 \\
(14.1 \%)\end{array}$ & $\begin{array}{c}0 \\
(0.0 \%) \\
\end{array}$ & $\begin{array}{c}207 \\
(41.6 \%) \\
\end{array}$ & $<0.001$ \\
\hline $\begin{array}{l}\text { Dental caries prevention } \\
\text { and control }\end{array}$ & $\begin{array}{c}11 \\
(40.7 \%)\end{array}$ & $\begin{array}{c}38 \\
(35.2 \%)\end{array}$ & $\begin{array}{c}42 \\
(36.2 \%)\end{array}$ & $\begin{array}{c}23 \\
(15.1 \%)\end{array}$ & $\begin{array}{c}7 \\
(8.2 \%)\end{array}$ & $\begin{array}{c}0 \\
(0.0 \%)\end{array}$ & $\begin{array}{c}121 \\
(24.3 \%)\end{array}$ & $<0.001$ \\
\hline $\begin{array}{l}\text { Dental health guidance } \\
\text { for individuals }\end{array}$ & $\begin{array}{c}10 \\
(37.0 \%)\end{array}$ & $\begin{array}{c}58 \\
(53.7 \%) *\end{array}$ & $\begin{array}{c}53 \\
(45.7 \%)\end{array}$ & $\begin{array}{c}58 \\
(38.2 \%)\end{array}$ & $\begin{array}{c}25 \\
(29.4 \%)\end{array}$ & $\begin{array}{c}0 \\
(0.0 \%) \\
\end{array}$ & $\begin{array}{c}204 \\
(41.0 \%)\end{array}$ & 0.001 \\
\hline $\begin{array}{l}\text { Dental health guidance } \\
\text { for groups }\end{array}$ & $\begin{array}{c}5 \\
(18.5 \%) \\
\end{array}$ & $\begin{array}{c}38 \\
(35.2 \%) \\
\end{array}$ & $\begin{array}{c}39 \\
(33.6 \%) \\
\end{array}$ & $\begin{array}{c}55 \\
(36.2 \%) \\
\end{array}$ & $\begin{array}{c}24 \\
(28.2 \%) \\
\end{array}$ & $\begin{array}{c}2 \\
(22.2 \%) \\
\end{array}$ & $\begin{array}{c}163 \\
(32.8 \%) \\
\end{array}$ & 0.433 \\
\hline $\begin{array}{l}\text { Handling of dental materials, } \\
\text { chairside assistant }\end{array}$ & $\begin{array}{c}9 \\
(33.3 \%)\end{array}$ & $\begin{array}{c}38 \\
(35.2 \%)\end{array}$ & $\begin{array}{c}39 \\
(33.6 \%)\end{array}$ & $\begin{array}{c}21 \\
(13.8 \%)\end{array}$ & $\begin{array}{c}3 \\
(3.5 \%)\end{array}$ & $\begin{array}{c}0 \\
(0.0 \%)\end{array}$ & $\begin{array}{c}110 \\
(22.1 \%)\end{array}$ & $<0.001$ \\
\hline $\begin{array}{l}\text { Chairside assistant tasks, such } \\
\text { as snap impression, polishing } \\
\text { of filling materials, } \\
\text { rubber dam, etc. }\end{array}$ & $\begin{array}{c}10 \\
(37.0 \%)\end{array}$ & $\begin{array}{c}39 \\
(36.1 \%)\end{array}$ & $\begin{array}{c}35 \\
(30.2 \%)\end{array}$ & $\begin{array}{c}17 \\
(11.2 \%)\end{array}$ & $\begin{array}{c}4 \\
(4.7 \%)\end{array}$ & $\begin{array}{c}0 \\
(0.0 \%)\end{array}$ & $\begin{array}{c}105 \\
(21.1 \%)\end{array}$ & $<0.001$ \\
\hline Nursing care & $\begin{array}{c}10 \\
(37.0 \%)\end{array}$ & $\begin{array}{c}44 \\
(40.7 \%)\end{array}$ & $\begin{array}{c}48 \\
(41.4 \%)\end{array}$ & $\begin{array}{c}58 \\
(38.2 \%)\end{array}$ & $\begin{array}{c}41 \\
(48.2 \%)\end{array}$ & $\begin{array}{c}5 \\
(55.6 \%)\end{array}$ & $\begin{array}{c}206 \\
(41.4 \%)\end{array}$ & 0.659 \\
\hline Counseling skills & $\begin{array}{c}13 \\
(48.1 \%)\end{array}$ & $\begin{array}{c}68 \\
(63.0 \%)\end{array}$ & $\begin{array}{c}85 \\
(73.3 \%)\end{array}$ & $\begin{array}{c}117 \\
(77 \%)\end{array}$ & $\begin{array}{c}58 \\
(68.2 \%)\end{array}$ & $\begin{array}{c}7 \\
(77.8 \%)\end{array}$ & $\begin{array}{c}348 \\
(70.0 \%)\end{array}$ & 0.022 \\
\hline Dysphagia rehabilitation & $\begin{array}{c}8 \\
(29.6 \%)\end{array}$ & $\begin{array}{c}66 \\
(61.1 \%)\end{array}$ & $\begin{array}{c}77 \\
(66.4 \%)\end{array}$ & $\begin{array}{c}102 \\
(67.1 \%)\end{array}$ & $\begin{array}{c}49 \\
(57.6 \%)\end{array}$ & $\begin{array}{c}5 \\
(55.6 \%)\end{array}$ & $\begin{array}{c}307 \\
(61.8 \%)\end{array}$ & 0.009 \\
\hline Myofunctional therapy & $\begin{array}{c}7 \\
(25.9 \%)\end{array}$ & $\begin{array}{c}31 \\
(28.7 \%)\end{array}$ & $\begin{array}{c}56 \\
(48.3 \%)\end{array}$ & $\begin{array}{c}71 \\
(46.7 \%)\end{array}$ & $\begin{array}{c}36 \\
(42.4 \%)\end{array}$ & $\begin{array}{c}5 \\
(55.6 \%)\end{array}$ & $\begin{array}{c}206 \\
(41.4 \%)\end{array}$ & 0.012 \\
\hline Other & $\begin{array}{c}1 \\
(3.7 \%)\end{array}$ & $\begin{array}{c}6 \\
(5.6 \%)\end{array}$ & $\begin{array}{c}12 \\
(10.3 \%)\end{array}$ & $\begin{array}{c}12 \\
(7.9 \%)\end{array}$ & $\begin{array}{c}6 \\
(7.1 \%)\end{array}$ & $\begin{array}{c}0 \\
(0.0 \%)\end{array}$ & $\begin{array}{c}37 \\
(7.4 \%)\end{array}$ & 0.652 \\
\hline
\end{tabular}


Table 1. Cont.

\begin{tabular}{|c|c|c|c|c|c|c|c|c|}
\hline Age & $20 \mathrm{~s}$ & $30 \mathrm{~s}$ & $40 \mathrm{~s}$ & $50 \mathrm{~s}$ & $60 \mathrm{~s}$ & $70 \mathrm{~s}$ & Total & $p$-Value \\
\hline \multicolumn{9}{|c|}{ If you were to start working again, how would you find a workplace? } \\
\hline Labor agency & $\begin{array}{c}23 \\
(69.7 \%)\end{array}$ & $\begin{array}{c}88 \\
(73.9 \%)\end{array}$ & $\begin{array}{c}71 \\
(74.1 \%)\end{array}$ & $\begin{array}{c}64 \\
(66.7 \%)\end{array}$ & $\begin{array}{c}21 \\
(56.8 \%)\end{array}$ & $\begin{array}{c}1 \\
(11.1 \%)\end{array}$ & $\begin{array}{c}268 \\
(70.1 \%)\end{array}$ & 0.349 \\
\hline Job magazines & $\begin{array}{c}12 \\
(36.4 \%)\end{array}$ & $\begin{array}{c}43 \\
(36.1 \%)\end{array}$ & $\begin{array}{c}29 \\
(30.2 \%)\end{array}$ & $\begin{array}{c}30 \\
(31.3 \%)\end{array}$ & $\begin{array}{c}11 \\
(29.7 \%)\end{array}$ & $\begin{array}{c}0 \\
(0.0 \%)\end{array}$ & $\begin{array}{c}125 \\
(32.6 \%)\end{array}$ & 0.803 \\
\hline $\begin{array}{l}\text { Recommendation from a } \\
\text { friend/acquaintance }\end{array}$ & $\begin{array}{c}15 \\
(45.5 \%) \\
\end{array}$ & $\begin{array}{c}61 \\
(51.3 \%) \\
\end{array}$ & $\begin{array}{c}40 \\
(41.7 \%) \\
\end{array}$ & $\begin{array}{c}43 \\
(44.8 \%)\end{array}$ & $\begin{array}{c}21 \\
(56.8 \%)\end{array}$ & $\begin{array}{c}1 \\
(11.1 \%)\end{array}$ & $\begin{array}{c}181 \\
(47.3 \%)\end{array}$ & 0.610 \\
\hline $\begin{array}{l}\text { Recommendation from dental } \\
\text { hygienist school }\end{array}$ & $\begin{array}{c}9 \\
(27.3 \%)\end{array}$ & $\begin{array}{c}22 \\
(18.5 \%)\end{array}$ & $\begin{array}{c}11 \\
(11.5 \%)\end{array}$ & $\begin{array}{c}9 \\
(9.4 \%)\end{array}$ & $\begin{array}{c}2 \\
(5.4 \%)\end{array}$ & $\begin{array}{c}0 \\
(0.0 \%)\end{array}$ & $\begin{array}{c}53 \\
(13.8 \%)\end{array}$ & 0.039 \\
\hline Internet & $\begin{array}{c}21 \\
(63.6 \%)\end{array}$ & $\begin{array}{c}72 \\
(60.5 \%)\end{array}$ & $\begin{array}{c}42 \\
(43.8 \%)\end{array}$ & $\begin{array}{c}36 \\
(37.5 \%)\end{array}$ & $\begin{array}{c}5 \\
(13.5 \%)\end{array}$ & $\begin{array}{c}0 \\
(0.0 \%)\end{array}$ & $\begin{array}{c}176 \\
(46.1 \%)\end{array}$ & $<0.001$ \\
\hline $\begin{array}{l}\text { Free referral by the Dental } \\
\text { Association and Dental } \\
\text { Hygienist Association }\end{array}$ & $\begin{array}{c}9 \\
(27.3 \%)\end{array}$ & $\begin{array}{c}27 \\
(22.7 \%)\end{array}$ & $\begin{array}{c}22 \\
(22.9 \%)\end{array}$ & $\begin{array}{c}37 \\
(38.5 \%)\end{array}$ & $\begin{array}{c}9 \\
(24.3 \%)\end{array}$ & $\begin{array}{c}1 \\
(11.1 \%)\end{array}$ & $\begin{array}{c}105 \\
(27.4 \%)\end{array}$ & 0.110 \\
\hline $\begin{array}{l}\text { Human resource } \\
\text { dispatch companies }\end{array}$ & $\begin{array}{c}2 \\
(6.1 \%)\end{array}$ & $\begin{array}{c}5 \\
(4.2 \%)\end{array}$ & $\begin{array}{c}15 \\
(15.6 \%)\end{array}$ & $\begin{array}{c}9 \\
(9.4 \%)\end{array}$ & $\begin{array}{c}1 \\
(2.7 \%)\end{array}$ & $\begin{array}{c}0 \\
(0.0 \%)\end{array}$ & $\begin{array}{c}32 \\
(8.4 \%)\end{array}$ & 0.044 \\
\hline Other & $\begin{array}{c}0 \\
(0.0 \%)\end{array}$ & $\begin{array}{c}3 \\
(2.5 \%)\end{array}$ & $\begin{array}{c}3 \\
(3.1 \%)\end{array}$ & $\begin{array}{c}5 \\
(5.2 \%)\end{array}$ & $\begin{array}{c}1 \\
(2.7 \%)\end{array}$ & $\begin{array}{c}0 \\
(0.0 \%)\end{array}$ & $\begin{array}{c}12 \\
(3.1 \%)\end{array}$ & 0.752 \\
\hline
\end{tabular}

* Statistically significant as determined by the log-linear analysis.

The main reasons for leaving a job were "Childbirth/child-rearing" $(16.9 \%)$ and "Relationship with the manager" (15.7\%). Leaving due to "Childbirth/child-rearing" was a statistically significant factor for the age groups younger than 49 . For those in their $20 \mathrm{~s}$ and 40s, "Childbirth/child-rearing" and "Relationship with the manager" were the main reasons for leaving a job. For those in their 30s, "Childbirth/child-rearing" (40.6\%) was the most frequent reason for leaving a job. For those in their 50s, the most frequent reason for leaving a job was "Interest in a different field" (18.2\%). For those in their 60s, the most frequent reason for leaving a job was "Personal health" (16.4\%). For those in their 70s, the most frequent reason for leaving a job was "Family matters" (18.1\%).

Regarding the item about factors that needed to be improved in the last workplace environment, 285 dental hygienists $(28.1 \%)$ answered "Interpersonal relationships at the workplace," which was the most common response, followed by "Improvement of salary (base up, regular pay raise, etc.,)" (25.5\%). These answers were statistically significant factors for individuals in all age groups except for those in their 70s. For those aged below 40 , the most frequent request was "Interpersonal relationships at the workplace." For those in their 50s, "Improvement of working conditions (base up, regular pay raise, etc.)" $(28.2 \%)$ and "Interpersonal relationships at the workplace" $(28.2 \%)$ were the most common answers. For those in their 60s, "Improvement of the working conditions (base up, regular pay raise, etc.,)" was the most frequent response. For those in their 70s, "Evaluation of expertise, qualification, etc.," (19.7\%) was the most frequent response.

The main obstacle to working again was "Working hours" (58.1\%). For respondents in their 20s, "My skills" (75.0\%) was a major obstacle. For those in their 30s and 40s, "Working hours" was a major obstacle. For those in their 50s and 60s, "Aging" presented a major obstacle. For those in their 70s, "Working hours" and "Aging" were major obstacles.

Approximately $48 \%$ of subjects stated that they wish to participate in an educational or upskill workshop to aid in workplace re-entry. "Counseling skills" was the program that attracted the greatest level of interest (70.0\%). "Dysphagia rehabilitation" was a highly requested program for all age groups except participants aged in their 20s. For those younger than 39, the "Scaling and route planning" program was frequently of interest. This was a statistically significant answer for respondents in their 20s, 30s, and 40s. For age groups older than 40 , "Counseling skills" was frequently of interest. 
In terms of finding a new workplace, approximately $70 \%$ of subjects stated that they would use a "Labor agency." For all generations, "Labor agency" was the most popular answer to this question. For age groups younger than 30," the number of subjects who answered "Internet" was notable.

A cross-tabulation showing participants' willingness to re-enter the workforce versus items related to leaving jobs and re-entry is shown in Table 2.

The main reason why dental hygienists who wished to re-enter the workforce had left their jobs was "Childbirth/child-rearing" (17.0\%). This reason was statistically significant related to the statement "I want to re-enter if conditions are satisfied."

For the question on improvements that should be made to their last workplace environment, $28.0 \%$ of subjects answered: "Interpersonal relationships at the workplace." This item was statistically significantly related to the response "I want to re-enter if conditions are satisfied." The response "I want to re-enter as soon as possible" was statistically significantly related to the answers "Interpersonal relationships at workplace," "Evaluation of expertise, qualifications, etc.," "Enhancement of medical safety system," "Ensuring employment stability," and "Enrichment of employee benefits".

In terms of obstacles to working again, $57.9 \%$ of subjects selected "Working hours." This item was identified as the major obstacle for subjects who answered "I want to reenter if conditions are satisfied." For the subjects who answered "I want to reenter as soon as possible," "My skills" was identified as the major obstacle.

Many subjects stated that they would be interested in participating in two lecture courses: "Dysphagia rehabilitation" (70.3\%) and "Myofunctional therapy" (61.7\%). Many of the subjects who answered "I want to re-enter as soon as possible" and "I want to re-enter if conditions are satisfied" selected these two lecture courses.

To find a new workplace, $70.1 \%$ of subjects stated that they would use a "Labor agency." The answers "Recommendation from a friend/acquaintance" and "Internet" were statistically significantly related to the response "I want to re-enter as soon as possible".

Table 2. Characteristics of the participants involved in this study by classification of willingness to re-enter.

\begin{tabular}{|c|c|c|c|c|c|c|}
\hline & $\begin{array}{l}\text { I Want to Re-Enter } \\
\text { As Soon As Possible }\end{array}$ & $\begin{array}{c}\text { I Want to Re-Enter if } \\
\text { Conditions Are Satisfied }\end{array}$ & $\begin{array}{l}\text { I Do Not Intend } \\
\text { to Re-Enter }\end{array}$ & I Do Not Know & Total & $p$-Value \\
\hline \multicolumn{7}{|c|}{ Why did you decide to leave the workplace where you were last working as a dental hygienist? } \\
\hline $\begin{array}{l}\text { Dissatisfaction with } \\
\text { work content }\end{array}$ & $\begin{array}{c}6 \\
(15 \%)\end{array}$ & $\begin{array}{c}41 \\
(11.6 \%)\end{array}$ & $\begin{array}{c}30 \\
(6.8 \%)\end{array}$ & $\begin{array}{c}20 \\
(10.6 \%)\end{array}$ & $\begin{array}{c}97 \\
(9.5 \%)\end{array}$ & 0.063 \\
\hline Payment/welfare & $\begin{array}{c}7 \\
(17.5 \%)\end{array}$ & $\begin{array}{c}63 \\
(17.9 \%)\end{array}$ & $\begin{array}{c}36 \\
(8.2 \%)\end{array}$ & $\begin{array}{c}28 \\
(14.9 \%)\end{array}$ & $\begin{array}{c}134 \\
(13.1 \%)\end{array}$ & $<0.001$ \\
\hline $\begin{array}{l}\text { Relationship with } \\
\text { the manager }\end{array}$ & $\begin{array}{c}12 \\
(30.0 \%)\end{array}$ & $\begin{array}{c}76 \\
(21.6 \%)\end{array}$ & $\begin{array}{c}43 \\
(9.8 \%)\end{array}$ & $\begin{array}{c}31 \\
(16.5 \%)\end{array}$ & $\begin{array}{c}162 \\
(15.9 \%)\end{array}$ & $<0.001$ \\
\hline Relationships with seniors & $\begin{array}{c}4 \\
(10 \%)\end{array}$ & $\begin{array}{c}31 \\
(8.8 \%)\end{array}$ & $\begin{array}{c}16 \\
(3.6 \%)\end{array}$ & $\begin{array}{c}12 \\
(6.4 \%)\end{array}$ & $\begin{array}{c}63 \\
(6.2 \%)\end{array}$ & 0.017 \\
\hline $\begin{array}{l}\text { Relationships with } \\
\text { colleagues }\end{array}$ & $\begin{array}{c}4 \\
(10 \%)\end{array}$ & $\begin{array}{c}34 \\
(9.7 \%)\end{array}$ & $\begin{array}{c}26 \\
(5.9 \%)\end{array}$ & $\begin{array}{c}15 \\
(8.0 \%)\end{array}$ & $\begin{array}{c}79 \\
(7.7 \%)\end{array}$ & 0.239 \\
\hline $\begin{array}{l}\text { Working status/ } \\
\text { working hours }\end{array}$ & $\begin{array}{c}8 \\
(20 \%)\end{array}$ & $\begin{array}{c}61 \\
(17.3 \%)\end{array}$ & $\begin{array}{c}33 \\
(7.5 \%)\end{array}$ & $\begin{array}{c}21 \\
(11.2 \%)\end{array}$ & $\begin{array}{c}123 \\
(12 \%)\end{array}$ & $<0.001$ \\
\hline $\begin{array}{l}\text { Long working } \\
\text { hours/overwork }\end{array}$ & $\begin{array}{c}6 \\
(15 \%)\end{array}$ & $\begin{array}{c}36 \\
(10.2 \%)\end{array}$ & $\begin{array}{c}23 \\
(5.2 \%)\end{array}$ & $\begin{array}{c}9 \\
(4.8 \%)\end{array}$ & $\begin{array}{c}74 \\
(7.2 \%)\end{array}$ & 0.006 \\
\hline Family matters & $\begin{array}{c}3 \\
(7.5 \%)\end{array}$ & $\begin{array}{c}39 \\
(11.1 \%)\end{array}$ & $\begin{array}{c}68 \\
(15.4 \%)\end{array}$ & $\begin{array}{c}21 \\
(11.2 \%)\end{array}$ & $\begin{array}{c}131 \\
(12.8 \%)\end{array}$ & 0.166 \\
\hline $\begin{array}{l}\text { Job transfer of } \\
\text { family member }\end{array}$ & $\begin{array}{c}0 \\
(0 \%)\end{array}$ & $\begin{array}{c}17 \\
(4.8 \%)\end{array}$ & $\begin{array}{c}8 \\
(1.8 \%)\end{array}$ & $\begin{array}{c}1 \\
(0.5 \%)\end{array}$ & $\begin{array}{c}26 \\
(2.5 \%)\end{array}$ & 0.006 \\
\hline Marriage & $\begin{array}{c}4 \\
(10.0 \%)\end{array}$ & $\begin{array}{c}47 \\
(13.4 \%)\end{array}$ & $\begin{array}{c}44 \\
(10.0 \%)\end{array}$ & $\begin{array}{c}23 \\
(12.2 \%)\end{array}$ & $\begin{array}{c}118 \\
(11.6 \%)\end{array}$ & 0.5 \\
\hline Childbirth/child-rearing & $\begin{array}{c}7 \\
(17.5 \%)\end{array}$ & $\begin{array}{c}93 \\
(26.4 \%) *\end{array}$ & $\begin{array}{c}41 \\
(9.3 \%) *\end{array}$ & $\begin{array}{c}33 \\
(17.6 \%)\end{array}$ & $\begin{array}{c}174 \\
(17.0 \%)\end{array}$ & $<0.001$ \\
\hline
\end{tabular}


Table 2. Cont.

\begin{tabular}{|c|c|c|c|c|c|c|}
\hline & $\begin{array}{l}\text { I Want to Re-Enter } \\
\text { As Soon As Possible }\end{array}$ & $\begin{array}{l}\text { I Want to Re-Enter if } \\
\text { Conditions Are Satisfied }\end{array}$ & $\begin{array}{l}\text { I Do Not Intend } \\
\text { to Re-Enter }\end{array}$ & I Do Not Know & Total & $p$-Value \\
\hline $\begin{array}{l}\text { Nursing care for } \\
\text { family member }\end{array}$ & $\begin{array}{c}1 \\
(2.5 \%)\end{array}$ & $\begin{array}{c}19 \\
(5.4 \%) *\end{array}$ & $\begin{array}{c}34 \\
(7.7 \%)^{*}\end{array}$ & $\begin{array}{c}24 \\
(12.8 \%)\end{array}$ & $\begin{array}{c}78 \\
(7.6 \%)\end{array}$ & 0.012 \\
\hline Personal health & $\begin{array}{c}3 \\
(7.5 \%)\end{array}$ & $\begin{array}{c}56 \\
(15.9 \%)\end{array}$ & $\begin{array}{c}58 \\
(13.2 \%)\end{array}$ & $\begin{array}{c}33 \\
(17.6 \%)\end{array}$ & $\begin{array}{c}150 \\
(14.7 \%)\end{array}$ & 0.248 \\
\hline $\begin{array}{l}\text { To improve the level of } \\
\text { work content }\end{array}$ & $\begin{array}{c}3 \\
(7.5 \%)\end{array}$ & $\begin{array}{c}13 \\
(3.7 \%)\end{array}$ & $\begin{array}{c}7 \\
(1.6 \%)\end{array}$ & $\begin{array}{c}5 \\
(2.7 \%)\end{array}$ & $\begin{array}{c}28 \\
(2.7 \%)\end{array}$ & 0.079 \\
\hline $\begin{array}{l}\text { Inadequate training } \\
\text { program }\end{array}$ & $\begin{array}{c}2 \\
(5 \%)\end{array}$ & $\begin{array}{c}18 \\
(5.1 \%) *\end{array}$ & $\begin{array}{c}7 \\
(1.6 \%)\end{array}$ & $\begin{array}{c}2 \\
(1.1 \%)\end{array}$ & $\begin{array}{c}29 \\
(2.8 \%)\end{array}$ & 0.008 \\
\hline Limits of personal skills & $\begin{array}{c}2 \\
(5 \%)\end{array}$ & $\begin{array}{c}10 \\
(2.8 \%) *\end{array}$ & $\begin{array}{c}31 \\
(7.0 \%)\end{array}$ & $\begin{array}{c}19 \\
(10.1 \%)\end{array}$ & $\begin{array}{c}62 \\
(6.1 \%)\end{array}$ & 0.006 \\
\hline $\begin{array}{l}\text { The job was } \\
\text { not rewarding }\end{array}$ & $\begin{array}{c}3 \\
(7.5 \%)\end{array}$ & $\begin{array}{c}27 \\
(7.7 \%)\end{array}$ & $\begin{array}{c}12 \\
(2.7 \%)\end{array}$ & $\begin{array}{c}9 \\
(4.8 \%)\end{array}$ & $\begin{array}{c}51 \\
(5.0 \%)\end{array}$ & 0.014 \\
\hline Interest in different field & $\begin{array}{c}2 \\
(5.0 \%)\end{array}$ & $\begin{array}{c}33 \\
(9.4 \%)\end{array}$ & $\begin{array}{c}51 \\
(11.6 \%)\end{array}$ & $\begin{array}{c}26 \\
(13.8 \%)\end{array}$ & $\begin{array}{c}112 \\
(11.0 \%)\end{array}$ & 0.250 \\
\hline Others & $\begin{array}{c}11 \\
(27.5 \%)\end{array}$ & $\begin{array}{c}88 \\
(25.0 \%)\end{array}$ & $\begin{array}{c}157 \\
(35.6 \%) *\end{array}$ & $\begin{array}{c}49 \\
(26.1 \%)\end{array}$ & $\begin{array}{c}305 \\
(29.9 \%)\end{array}$ & 0.006 \\
\hline \multicolumn{7}{|c|}{ What do you think should have been improved about your workplace where you were working last? } \\
\hline $\begin{array}{l}\text { Improvement of working } \\
\text { conditions (base up, } \\
\text { regular pay raise, etc.) }\end{array}$ & $\begin{array}{c}15 \\
(37.5 \%)\end{array}$ & $\begin{array}{c}102 \\
(29.1 \%)\end{array}$ & $\begin{array}{c}87 \\
(20.5 \%) *\end{array}$ & $\begin{array}{c}52 \\
(28.6 \%)\end{array}$ & $\begin{array}{c}256 \\
(25.7 \%)\end{array}$ & 0.007 \\
\hline Reduction of work load & $\begin{array}{c}6 \\
(15.0 \%) \\
\end{array}$ & $\begin{array}{c}62 \\
(17.7 \%) \\
\end{array}$ & $\begin{array}{c}48 \\
(11.3 \%) \\
\end{array}$ & $\begin{array}{c}30 \\
(16.5 \%) \\
\end{array}$ & $\begin{array}{c}146 \\
(14.6 \%) \\
\end{array}$ & 0.073 \\
\hline $\begin{array}{l}\text { Interpersonal } \\
\text { relationships at } \\
\text { the workplace }\end{array}$ & $\begin{array}{c}15 \\
(37.5 \%)\end{array}$ & $\begin{array}{c}128 \\
(36.6 \%)\end{array}$ & $\begin{array}{c}88 \\
(20.7 \%)\end{array}$ & $\begin{array}{c}48 \\
(26.4 \%)\end{array}$ & $\begin{array}{c}279 \\
(28.0 \%)\end{array}$ & $<0.001$ \\
\hline $\begin{array}{l}\text { Reduction of the } \\
\text { working load }\end{array}$ & $\begin{array}{c}6 \\
(15.0 \%)\end{array}$ & $\begin{array}{c}69 \\
(19.7 \%)\end{array}$ & $\begin{array}{c}43 \\
(10.1 \%)\end{array}$ & $\begin{array}{c}25 \\
(13.7 \%)\end{array}$ & $\begin{array}{c}143 \\
(14.3 \%)\end{array}$ & 0.002 \\
\hline Taking a vacation & $\begin{array}{c}4 \\
(10.0 \%)\end{array}$ & $\begin{array}{c}78 \\
(22.3 \%)\end{array}$ & $\begin{array}{c}64 \\
(15.1 \%)\end{array}$ & $\begin{array}{c}35 \\
(19.2 \%)\end{array}$ & $\begin{array}{c}181 \\
(18.2 \%)\end{array}$ & 0.034 \\
\hline $\begin{array}{l}\text { Enhancement of } \\
\text { child-rearing support }\end{array}$ & $\begin{array}{c}2 \\
(5.0 \%)\end{array}$ & $\begin{array}{c}43 \\
(12.3 \%)\end{array}$ & $\begin{array}{c}16 \\
(3.8 \%)\end{array}$ & $\begin{array}{c}13 \\
(7.1 \%)\end{array}$ & $\begin{array}{c}74 \\
(7.4 \%)\end{array}$ & $<0.001$ \\
\hline $\begin{array}{l}\text { Enhancement of nursing } \\
\text { care support }\end{array}$ & $\begin{array}{c}1 \\
(2.5 \%)\end{array}$ & $\begin{array}{c}4 \\
(1.1 \%) *\end{array}$ & $\begin{array}{c}10 \\
(2.4 \%)\end{array}$ & $\begin{array}{c}9 \\
(4.9 \%)\end{array}$ & $\begin{array}{c}24 \\
(2.4 \%)\end{array}$ & 0.061 \\
\hline $\begin{array}{l}\text { Evaluation of expertise, } \\
\text { qualifications, etc. }\end{array}$ & $\begin{array}{c}9 \\
(22.5 \%) *\end{array}$ & $\begin{array}{c}66 \\
(18.9 \%) *\end{array}$ & $\begin{array}{c}64 \\
(15.1 \%)\end{array}$ & $\begin{array}{c}36 \\
(19.8 \%)\end{array}$ & $\begin{array}{c}175 \\
(17.6 \%)\end{array}$ & 0.316 \\
\hline $\begin{array}{l}\text { Enriching opportunities to } \\
\text { improve personal skills } \\
\text { through education, } \\
\text { training, etc. }\end{array}$ & $\begin{array}{c}9 \\
(22.5 \%)\end{array}$ & $\begin{array}{c}66 \\
(18.9 \%)\end{array}$ & $\begin{array}{c}51 \\
(12.0 \%)\end{array}$ & $\begin{array}{c}14 \\
(7.7 \%)\end{array}$ & $\begin{array}{c}140 \\
(14 \%)\end{array}$ & 0.001 \\
\hline $\begin{array}{c}\text { Introduction of various } \\
\text { work styles and } \\
\text { working hours }\end{array}$ & $\begin{array}{c}4 \\
(10.0 \%)\end{array}$ & $\begin{array}{c}38 \\
(10.9 \%)\end{array}$ & $\begin{array}{c}2 \\
4(5.6 \%)\end{array}$ & $\begin{array}{c}17 \\
(9.3 \%)\end{array}$ & $\begin{array}{c}83 \\
(8.3 \%)\end{array}$ & 0.062 \\
\hline $\begin{array}{l}\text { Enhancement of the } \\
\text { medical safety system }\end{array}$ & $\begin{array}{c}6 \\
(15.0 \%) * \\
\end{array}$ & $\begin{array}{c}36 \\
(10.3 \%) *\end{array}$ & $\begin{array}{c}25 \\
(5.9 \%) \\
\end{array}$ & $\begin{array}{c}9 \\
(4.9 \%) \\
\end{array}$ & $\begin{array}{c}76 \\
(7.6 \%)\end{array}$ & 0.016 \\
\hline $\begin{array}{l}\text { Ensuring employment } \\
\text { stability }\end{array}$ & $\begin{array}{c}9 \\
(22.5 \%) *\end{array}$ & $\begin{array}{c}39 \\
(11.1 \%)\end{array}$ & $\begin{array}{c}23 \\
(5.4 \%)\end{array}$ & $\begin{array}{c}14 \\
(7.7 \%)\end{array}$ & $\begin{array}{c}85 \\
(8.5 \%)\end{array}$ & $<0.001$ \\
\hline $\begin{array}{c}\text { Enrichment of } \\
\text { employee benefits }\end{array}$ & $\begin{array}{c}11 \\
(27.5 \%) *\end{array}$ & $\begin{array}{c}61 \\
(17.4 \%) \\
\end{array}$ & $\begin{array}{c}57 \\
(13.4 \%) \\
\end{array}$ & $\begin{array}{c}24 \\
(13.2 \%) \\
\end{array}$ & $\begin{array}{c}153 \\
(15.3 \%) \\
\end{array}$ & 0.055 \\
\hline Nothing in particular & $\begin{array}{c}8 \\
(20.0 \%)\end{array}$ & $\begin{array}{c}45 \\
(12.9 \%) *\end{array}$ & $\begin{array}{c}136 \\
(32.0 \%)\end{array}$ & $\begin{array}{c}53 \\
(29.1 \%)\end{array}$ & $\begin{array}{c}242 \\
(24.3 \%)\end{array}$ & $<0.001$ \\
\hline Other & $\begin{array}{c}3 \\
(7.5 \%)\end{array}$ & $\begin{array}{c}34 \\
(9.7 \%)\end{array}$ & $\begin{array}{c}30 \\
(7.1 \%)\end{array}$ & $\begin{array}{c}12 \\
(6.6 \%)\end{array}$ & $\begin{array}{c}79 \\
(7.9 \%)\end{array}$ & 0.489 \\
\hline
\end{tabular}


Table 2. Cont

\begin{tabular}{|c|c|c|c|c|c|c|}
\hline & $\begin{array}{l}\text { I Want to Re-Enter } \\
\text { As Soon As Possible }\end{array}$ & $\begin{array}{l}\text { I Want to Re-Enter if } \\
\text { Conditions Are Satisfied }\end{array}$ & $\begin{array}{l}\text { I Do Not Intend } \\
\text { to Re-Enter }\end{array}$ & I Do Not Know & Total & $p$-Value \\
\hline \multicolumn{7}{|c|}{ Are there any obstacles that are making it difficult for you to start working again as a dental hygienist? ("Yes") } \\
\hline Yes & $\begin{array}{c}24 \\
(66.7 \%)^{*}\end{array}$ & $\begin{array}{c}315 \\
(89.5 \%)\end{array}$ & 0 & 0 & $\begin{array}{c}339 \\
(87.4 \%)\end{array}$ & $<0.001$ \\
\hline \multicolumn{7}{|c|}{ What are these obstacles? } \\
\hline Salary & $\begin{array}{c}10 \\
(41.7 \%)\end{array}$ & $\begin{array}{c}74 \\
(23.8 \%)\end{array}$ & & & $\begin{array}{c}84 \\
(25.1 \%)\end{array}$ & 0.052 \\
\hline Working hours & $\begin{array}{c}11 \\
(45.8 \%)\end{array}$ & $\begin{array}{c}183 \\
(58.8 \%)\end{array}$ & & & $\begin{array}{c}194 \\
(57.9 \%)\end{array}$ & 0.214 \\
\hline My skills & $\begin{array}{c}13 \\
(54.2 \%)\end{array}$ & $\begin{array}{c}147 \\
(47.3 \%)\end{array}$ & & & $\begin{array}{c}160 \\
(47.8 \%)\end{array}$ & 0.514 \\
\hline No consultation & $\begin{array}{c}4 \\
(16.7 \%) \\
\end{array}$ & $\begin{array}{c}24 \\
(7.7 \%)\end{array}$ & & & $\begin{array}{c}28 \\
(8.4 \%)\end{array}$ & 0.127 \\
\hline Aging & $\begin{array}{c}7 \\
(29.2 \%) \\
\end{array}$ & $\begin{array}{c}86 \\
(27.7 \%) \\
\end{array}$ & & & $\begin{array}{c}93 \\
(27.8 \%) \\
\end{array}$ & 0.873 \\
\hline Others & $\begin{array}{c}8 \\
(33.3 \%)\end{array}$ & $\begin{array}{c}89 \\
(28.6 \%) \\
\end{array}$ & & & $\begin{array}{c}97 \\
(29.0 \%)\end{array}$ & 0.624 \\
\hline \multicolumn{7}{|c|}{ Would you want to attend a lecture-based course for re-entry? ("Yes") } \\
\hline Yes & $\begin{array}{c}28 \\
(70.0 \%)\end{array}$ & $\begin{array}{c}285 \\
(81.7 \%)\end{array}$ & $\begin{array}{c}64 \\
(14.7 \%)\end{array}$ & $\begin{array}{c}111 \\
(59.7 \%)\end{array}$ & $\begin{array}{c}488 \\
(48.3 \%)\end{array}$ & $<0.001$ \\
\hline \multicolumn{7}{|c|}{ What kind of lecture courses would you want to attend? } \\
\hline $\begin{array}{l}\text { Professional oral } \\
\text { care techniques }\end{array}$ & $\begin{array}{c}16 \\
(57.1 \%) \\
\end{array}$ & $\begin{array}{c}135 \\
(47.4 \%) \\
\end{array}$ & $\begin{array}{c}21 \\
(32.8 \%) *\end{array}$ & $\begin{array}{c}54 \\
(48.6 \%) \\
\end{array}$ & $\begin{array}{c}226 \\
(46.3 \%) \\
\end{array}$ & 0.094 \\
\hline $\begin{array}{l}\text { Scaling and } \\
\text { route planning }\end{array}$ & $\begin{array}{c}14 \\
(50 \%)\end{array}$ & $\begin{array}{c}136 \\
(47.7 \%) \\
\end{array}$ & $\begin{array}{c}14 \\
(21.9 \%) *\end{array}$ & $\begin{array}{c}43 \\
(38.7 \%) \\
\end{array}$ & $\begin{array}{c}207 \\
(42.4 \%)\end{array}$ & 0.001 \\
\hline $\begin{array}{l}\text { Dental caries prevention } \\
\text { and control }\end{array}$ & $\begin{array}{c}10 \\
(35.7 \%)\end{array}$ & $\begin{array}{c}72 \\
(25.3 \%)\end{array}$ & $\begin{array}{c}10 \\
(15.6 \%)\end{array}$ & $\begin{array}{c}31 \\
(27.9 \%)\end{array}$ & $\begin{array}{c}123 \\
(25.2 \%)\end{array}$ & 0.158 \\
\hline $\begin{array}{l}\text { Dental health guidance } \\
\text { for individuals }\end{array}$ & $\begin{array}{c}13 \\
(46.4 \%)\end{array}$ & $\begin{array}{c}125 \\
(43.9 \%)\end{array}$ & $\begin{array}{c}17 \\
(26.6 \%)^{*}\end{array}$ & $\begin{array}{c}50 \\
(45.0 \%)\end{array}$ & $\begin{array}{c}205 \\
(42.0 \%)\end{array}$ & 0.063 \\
\hline $\begin{array}{l}\text { Dental health guidance } \\
\text { for group }\end{array}$ & $\begin{array}{c}11 \\
(39.3 \%)\end{array}$ & $\begin{array}{c}95 \\
(33.3 \%)\end{array}$ & $\begin{array}{c}15 \\
(23.4 \%)\end{array}$ & $\begin{array}{c}41 \\
(36.9 \%)\end{array}$ & $\begin{array}{c}162 \\
(33.2 \%)\end{array}$ & 0.270 \\
\hline $\begin{array}{c}\text { Handling of } \\
\text { dental materials, } \\
\text { chairside assistant }\end{array}$ & $\begin{array}{c}5 \\
(17.9 \%)\end{array}$ & $\begin{array}{c}70 \\
(24.6 \%)\end{array}$ & $\begin{array}{c}5 \\
(7.8 \%) *\end{array}$ & $\begin{array}{c}31 \\
(27.9 \%)\end{array}$ & $\begin{array}{c}111 \\
(22.7 \%)\end{array}$ & 0.013 \\
\hline $\begin{array}{l}\text { Chairside assistant } \\
\text { techniques, e.g., snap } \\
\text { impression, polishing of } \\
\text { filling materials, rubber } \\
\text { dam, etc. }\end{array}$ & $\begin{array}{c}5 \\
(17.9 \%)\end{array}$ & $\begin{array}{c}69 \\
(24.2 \%)\end{array}$ & $\begin{array}{c}7 \\
(10.9 \%)\end{array}$ & $\begin{array}{c}26 \\
(23.4 \%)\end{array}$ & $\begin{array}{c}107 \\
(21.9 \%)\end{array}$ & 0.122 \\
\hline Counseling skills & $\begin{array}{c}14 \\
(50.0 \%)\end{array}$ & $\begin{array}{c}116 \\
(40.7 \%) \\
\end{array}$ & $\begin{array}{c}28 \\
(43.8 \%) \\
\end{array}$ & $\begin{array}{c}47 \\
(42.3 \%) \\
\end{array}$ & $\begin{array}{c}205 \\
(42.0 \%) \\
\end{array}$ & 0.797 \\
\hline Dysphagia rehabilitation & $\begin{array}{c}22 \\
(78.6 \%)\end{array}$ & $\begin{array}{c}195 \\
(68.4 \%)\end{array}$ & $\begin{array}{c}45 \\
(70.3 \%) \\
\end{array}$ & $\begin{array}{c}81 \\
(73.0 \%) \\
\end{array}$ & $\begin{array}{c}343 \\
(70.3 \%)\end{array}$ & 0.620 \\
\hline Myofunctional therapy & $\begin{array}{c}19 \\
(67.9 \%)\end{array}$ & $\begin{array}{c}175 \\
(61.4 \%)\end{array}$ & $\begin{array}{c}35 \\
(54.7 \%) \\
\end{array}$ & $\begin{array}{c}72 \\
(64.9 \%)\end{array}$ & $\begin{array}{c}301 \\
(61.7 \%)\end{array}$ & 0.520 \\
\hline Nursing care skills & $\begin{array}{c}9 \\
(32.1 \%)\end{array}$ & $\begin{array}{c}111 \\
(38.9 \%)\end{array}$ & $\begin{array}{c}27 \\
(42.2 \%)\end{array}$ & $\begin{array}{c}54 \\
(48.6 \%)\end{array}$ & $\begin{array}{c}201 \\
(41.2 \%)\end{array}$ & 0.249 \\
\hline Other & $\begin{array}{c}1 \\
(3.6 \%)\end{array}$ & $\begin{array}{c}22 \\
(7.7 \%)\end{array}$ & $\begin{array}{c}4 \\
(6.3 \%)\end{array}$ & $\begin{array}{c}9 \\
(8.1 \%)\end{array}$ & $\begin{array}{c}36 \\
(7.4 \%)\end{array}$ & 0.838 \\
\hline
\end{tabular}


Table 2. Cont.

\begin{tabular}{|c|c|c|c|c|c|}
\hline & $\begin{array}{l}\text { I Want to Re-Enter } \\
\text { As Soon As Possible }\end{array}$ & $\begin{array}{l}\text { I Want to Re-Enter if } \\
\text { Conditions Are Satisfied }\end{array}$ & $\begin{array}{l}\text { I Do Not Intend } \\
\text { to Re-Enter }\end{array}$ & Total & $p$-Value \\
\hline \multicolumn{6}{|c|}{ If you were to start working again, how would you find a workplace? } \\
\hline Labor agency & $\begin{array}{c}27 \\
(75.1 \%)\end{array}$ & $\begin{array}{c}243 \\
(69.61 \%)\end{array}$ & & $\begin{array}{c}270 \\
(70.1 \%)\end{array}$ & 0.502 \\
\hline Job magazines & $\begin{array}{c}9 \\
(25.1 \%)\end{array}$ & $\begin{array}{c}117 \\
(33.5 \%) \\
\end{array}$ & & $\begin{array}{c}126 \\
(32.7 \%)\end{array}$ & 0.299 \\
\hline $\begin{array}{l}\text { Recommendation from a } \\
\text { friend/acquaintance }\end{array}$ & $\begin{array}{c}5 \\
(13.9 \%) *\end{array}$ & $\begin{array}{c}177 \\
(50.7 \%)\end{array}$ & & $\begin{array}{c}182 \\
(47.3 \%)\end{array}$ & $<0.001$ \\
\hline $\begin{array}{l}\text { Recommendation from } \\
\text { dental hygienist school }\end{array}$ & $\begin{array}{c}2 \\
(5.6 \%)\end{array}$ & $\begin{array}{c}51 \\
(14.6 \%) \\
\end{array}$ & & $\begin{array}{c}53 \\
(13.8 \%) \\
\end{array}$ & 0.133 \\
\hline Internet & $\begin{array}{c}24 \\
(66.7 \%) * \\
\end{array}$ & $\begin{array}{c}154 \\
(44.1 \%)\end{array}$ & & $\begin{array}{c}178 \\
(46.2 \%) \\
\end{array}$ & 0.010 \\
\hline $\begin{array}{l}\text { Free referral by the Dental } \\
\text { Association and Dental } \\
\text { Hygienist Association }\end{array}$ & $\begin{array}{c}7 \\
(19.4 \%)\end{array}$ & $\begin{array}{c}98 \\
(28.1 \%)\end{array}$ & & $\begin{array}{c}105 \\
(27.3 \%)\end{array}$ & 0.268 \\
\hline $\begin{array}{l}\text { Human resource } \\
\text { dispatch companies }\end{array}$ & $\begin{array}{c}4 \\
(11.1 \%)\end{array}$ & $\begin{array}{c}28 \\
(81.0 \%)\end{array}$ & & $\begin{array}{c}32 \\
(8.3 \%)\end{array}$ & 0.523 \\
\hline Other & $\begin{array}{c}2 \\
(5.6 \%)\end{array}$ & $\begin{array}{c}10 \\
(2.9 \%)\end{array}$ & & $\begin{array}{c}12 \\
(3.1 \%)\end{array}$ & 0.376 \\
\hline
\end{tabular}

* Statistically significant cell according to the log-linear analysis.

The cross-tabulation of the items investigated in this study against age groups was illustrated graphically by correspondence analysis. Figure 1 shows the biplot of the willingness to re-enter and reasons for leaving jobs. "Working status/working hours" is located near to subjects who answered "I want to re-enter as soon as possible." The item "Childbirth/child-rearing" is located near to "I want to re-enter if conditions are satisfied." "Family matters" and "Personal health" are in close proximity to "I do not intend to" .

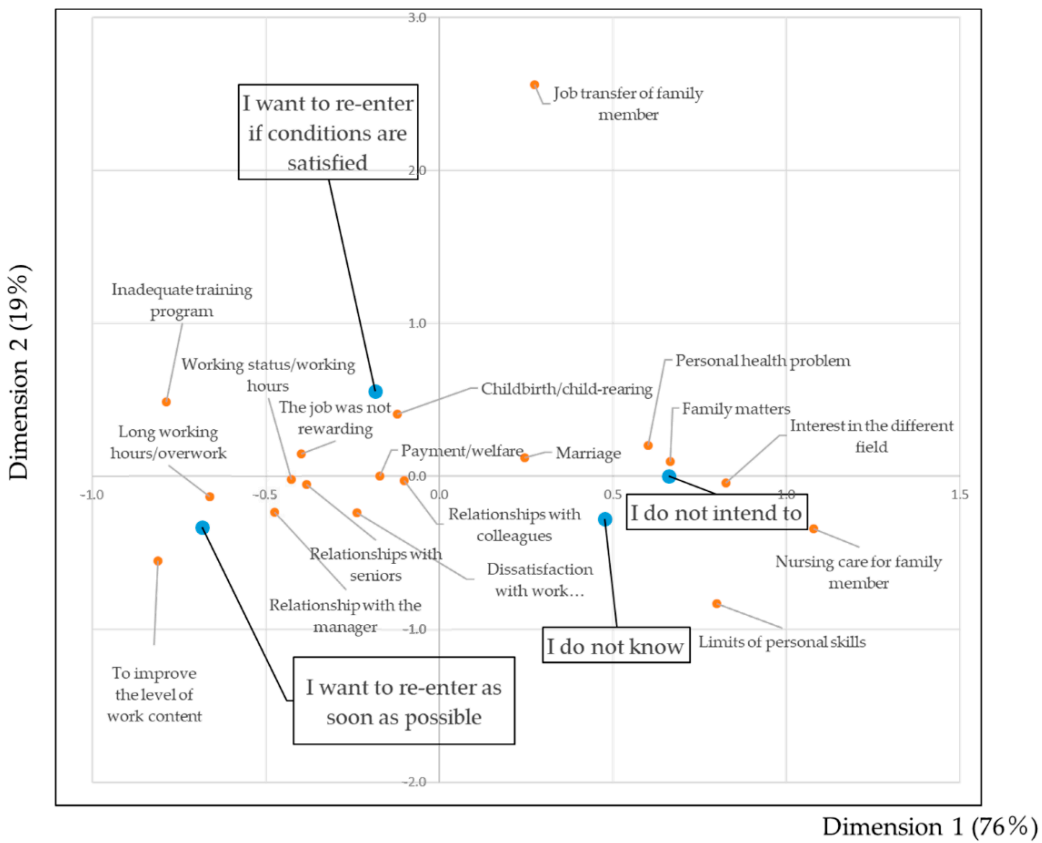

Figure 1. Biplot diagram showing the willingness to re-enter and the reasons for leaving jobs. The blue circles indicate the level of willingness to re-enter: "I want to re-enter as soon as possible," "I want to re-enter if conditions are satisfied," "I do not intend to," and "I do not know." The red circles show 18 reasons for leaving jobs. The closer the items are to each other, the higher the association between items. 
Figure 2 shows a biplot of the willingness to re-enter and the need for improvement of the workplace environment. "Enrichment of employee benefits" is located near to the item "I want to re-enter as soon as possible." "Reduction of the working load" is located near to the item "I want to reenter if conditions are satisfied." "Evaluation of expertise, qualifications, etc.," and "Enhancement of nursing care support" are in close proximity to "I do not intend to."

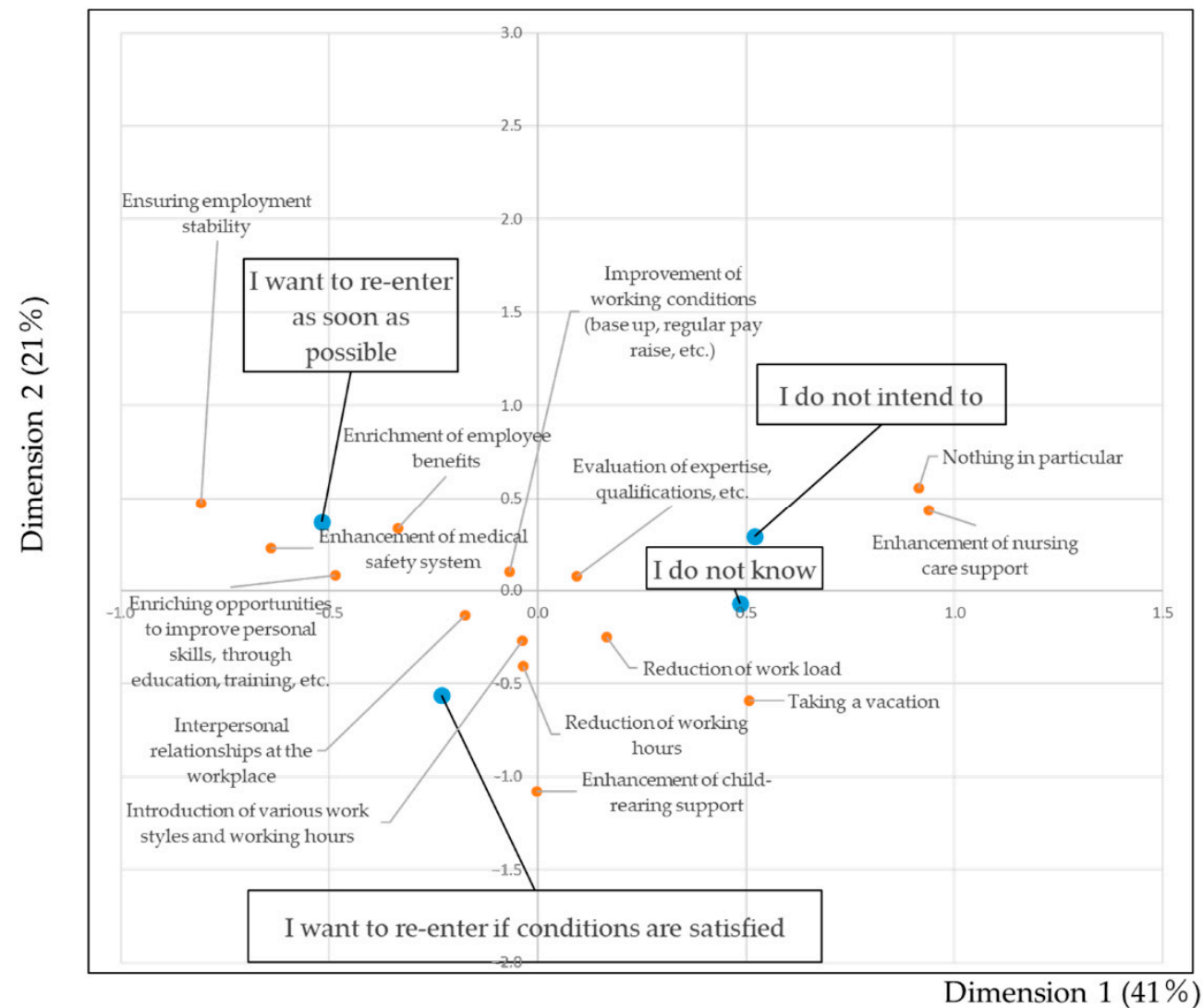

Figure 2. Biplot showing the willingness to re-enter and the need for improvement of the workplace environment. The blue circles show the willingness to re-enter. The red circles show 14 items related to improvement of the workplace environment. The closer the items are, the greater the association between items.

\section{Discussion}

The Japan Dental Hygienists' Association surveys the working conditions of dental hygienists every five years. In this survey, dental hygienists who had left their jobs were included. A report of this survey was presented on the homepage of the Japan Dental Hygienists' Association [12]. One of the aims of this survey is to support the re-entry of dormant dental hygienists into the workplace. Therefore, the questionnaire contained items concerning factors associated with a leaving job, factors that would support workplace re-entry, and factors associated with improving the workplace environment. However, this report only presents descriptive statistics. A more in-depth analysis including statistical modeling is indispensable to address obstacles to the willingness to re-enter the workforce and to determine the effects of factors on the willingness to re-enter. To address this issue, we applied a log-linear analysis and correspondence analysis to identify the statistically significant factors related to the willingness to re-enter the workforce.

By Japanese dental hygienist law, only women can be licensed dental hygienists. The law was amended in 2015, and since then, men have also been allowed to be licensed as dental hygienists. However, the number of dental hygienists is still at a low level. 
More than approximately $40 \%$ of dental hygienists surveyed stated that they do not wish to reenter the dental hygienist workforce. The social position of dental hygienists needs to improve and should be reflect by salaries that are commensurate with the work involved. On the other hand, around $40 \%$ of dormant dental hygienists stated that they would like to re-enter the workforce. Encouraging dormant dental hygienists to re-enter the workforce is an effective strategy to resolve the supply-demand imbalance. Many dental hygienists who stated that they would like to re-enter feel that there are obstacles to re-entry. Therefore, analyzing the reasons for leaving jobs, obstacles to reentry, and ways to improve the working environment is essential, especially to encourage dental hygienists who wish to reenter the workforce immediately.

The major reason reported for leaving a job was "Childbirth/child-rearing." These respondents stated that they would like to re-enter the workforce if conditions are improved. The log-linear analysis indicated that "Childbirth/child-rearing" was a statistically significant factor for the younger generation (those aged less than 49 years old). In addition, this item was statistically significantly related to the response "I want to reenter if conditions are satisfied." The correspondence analysis revealed that "Childbirth/child-rearing" is the most important factor that needs to be addressed to entice dormant dental hygienists to return to work.

"Relationship with the manager" was the second most common reason given for leaving a job. Some dental hygienists stated that they had left jobs due to human relationships with seniors or colleagues. More than half of them wished to re-enter.

In terms of improving the working environment, "Improvement of working condition (base up, regular pay raise, etc.,)" and "Interpersonal relationships in the workplace" had high response rates. The log-linear analysis indicated that the item "Improvement of working conditions (base up, regular pay raise, etc.,)" was a statistically significant response for all generations, except for participants in their 70s. Under the Japanese medical insurance system, each dental practice gives fixed pay. Japanese dental hygienist law regulates the practice of dental hygienists, requiring the instruction of a dentist. The salary of a dental hygienist depends on the interests of the dental office [13]. Further study on the cost-effectiveness of the employment of dental hygienists is necessary. The log-linear analysis indicated that the item "Interpersonal relationships at the workplace" was a statistically significant factor for all generations, except for respondents in their 70s. Moreover, this item was found to be statistically significantly associated with the response "I want to re-enter if conditions are satisfied." This indicates that good human relationships are the basis of a good working environment. In Japan, the dental service supply mainly depends on private dental clinics, and most of them operate on a small scale. Most of the dental clinics are managed by dentists. It is hard to improve human relationships in private dental clinics because of the limited resources available. One solution is to implement systematic construction of the community through a dental association or dental hygienist association. However, the improvement of human relationships is a complicated task. Further study on improving human relationships is necessary.

When focused on respondents in their 30s, "Enhancement of child-rearing support" and "Introduction of various work style and working hours" were shown to be statistically significant factors. Considering that a frequent reason for leaving a job was "Childbirth/child-rearing," flexible work arrangements are needed. Unfortunately, most dental clinics in Japan operate on a small scale and are privately managed. The number of dental hygienists is not sufficient. It is currently hard to introduce a flexible working system. Therefore, a social support system is necessary to resolve this issue. The Japanese government has been developing policy measures to improve the working environment for women. Several points need to be improved, including working hours. These factors are not unique to Japan. Similar problems occur in other parts of the world [14-16].

Frequent obstacles to re-entry were "Working hours," "My skills," and "salary." For respondents under the age of 50, more than $70 \%$ of dental hygienists recognized working hours as being an obstacle. This result has been found in previous studies in Japan [13-16]. 
Most Japanese hygienists work in private dental clinics. Many of these clinics are open until 8:00 or 9:00 pm. Some of them are also open on weekends and holidays. Given the situation, dental hygienists may have complaints about the working hours.

"My skills" was also cited as a frequent obstacle. For respondents in their 20s, 75\% recognized their skills as being an obstacle. For respondents in their $60 \mathrm{~s}, 32.4 \%$ recognized skills as being an obstacle. Moreover, for the subjects who answered "I want to reenter as soon as possible," "My skills" was a statistically significant response. Many of the subjects stated that they would like to participate in a lecture-based course for reentry. Many of the subjects who answered "I want to re-enter as soon as possible" and "I want to re-enter if conditions are satisfied" selected two lecture courses as being of interest: "Dysphagia rehabilitation" and "Myofunctional therapy." The results showed a different tendency to previous surveys $[17,18]$. In particular, a survey of the working environment of Japanese dental hygienists for the fiscal year 2009 reported that participates were interested in "Scaling and route planning" more than "Myofunctional therapy" or "Dysphagia rehabilitation." According to the Report on Public Health Administration and Services [4], 1.0\% of dental hygienists were working in welfare facilities for the elderly. The number of dental hygienists working in welfare facilities for older people has been increasing year by year: $0.2 \%$ in $2010,0.3 \%$ in $2012,0.4$ in 2014, and $0.8 \%$ in 2016. Since Japan is a super-aging society, it is necessary for dental hygienists to be aware of the demands of oral health management for older adults [19]. This may reflect the need for dental hygienists to conduct home-based dental care for older adults.

More than $40 \%$ of the subjects stated that they would be interested in courses on scaling root plaining and professional oral care techniques. More than $77 \%$ of subjects in their 20s and more than half of those in their 40s gave this response. Dental hygienists may recognize these fundamental skills as being a must for working as dental professionals. On the contrary, "limits of personal skills" was not stated as a major reason for leaving a job. Removing anxiety related to skills may promote re-entry. Further improvement of the skill-up system is necessary. Respondents in the younger age group $(\leq 29)$ stated that they would be interested in receiving a lecture on "Enriching opportunities to improve personal skills, through education, training, etc." Most dental hygienist schools in Japan involve a 3-year diploma program. In most cases, working as a dental hygienist involves long working hours. There are limited opportunities to participate in postgraduate education or training programs.

There are several limitations to this study. First, the study population was limited to the members of the Japan Dental Hygienists Association. Second, the data were collected from individuals who self-enrolled in the study. Third, the data were self-reported, and the subjects' actual behaviors were not observed. In addition, not all subjects answered all of the questions.

\section{Conclusions}

Dental clinics in Japan are experiencing a shortage of dental hygienists. Reintroducing dormant dental hygienists into the workforce would be an effective strategy to resolve the supply-demand imbalance. For the re-entry of dormant dental hygienists, the working environment needs to be improved. The introduction of a flexible working system and training programs focused on re-entry as well as the improvement of human relationships may be key factors.

Supplementary Materials: The following are available online at https:/ /www.mdpi.com/1660-460 1/18/4/2049/s1 , File 1: Questionnaire analyzed in this study.

Author Contributions: Conceptualization, Y.N., Y.O., and N.T.; methodology, Y.N., Y.O., and N.T.; software, Y.N.; validation, Y.N., Y.O., and N.T.; formal analysis, Y.N.; investigation, Y.N., Y.O., and N.T.; resources, Y.O. and N.T.; data curation, N.Y.; writing-original draft preparation, A.O.; writingreview and editing, Y.N., Y.O., and Y.Y.; visualization, Y.N.; supervision, N.H. (Noriyasu Hosoya), 
N.H. (Nobuhiro Hanada), and N.T.; project administration, N.T. All authors have read and agreed to the published version of the manuscript.

Funding: This study was supported by JSPS KAKENHI (grant numbers 17K12030, 20K10303), the SECOM Science and Technology Foundation, and the annual fund of the JAPAN DENTAL HYGIENISTS' ASSOCIATION.

Institutional Review Board Statement: The study was conducted according to the guidelines of the Declaration of Helsinki and approved by the Ethics Committee of Tsurumi University School of Dental Medicine (approval number: 1837).

Informed Consent Statement: Informed consent was obtained from all subjects involved in the study.

Data Availability Statement: Data are available from the corresponding author on reasonable request.

Conflicts of Interest: The authors declare no conflict of interest.

\section{References}

1. Population Estimates, Statistics Bureau, Ministry of Internal Affairs and Communications Japan. Available online: https://https: / /www.stat.go.jp/english/data/jinsui/tsuki/index.html (accessed on 1 December 2020).

2. For Achievement of the Zero Children Waiting List, Ministry of Internal Affairs and Communication Japan. Available online: https://www.mhlw.go.jp/english/policy/children/children-childrearing/dl/150407-03.pdf (accessed on 1 December 2020).

3. Labour Force Survey, Statistics Bureau, Ministry of Internal Affairs and Communications Japan. Available online: https: //www.stat.go.jp/data/roudou/sokuhou/tsuki/pdf/gaiyou.pdf (accessed on 1 December 2020).

4. Vital Statistics, Ministry of Health, Labor and Welfare Japan. Available online: https://www.mhlw.go.jp/toukei/saikin/hw/ jinkou/kakutei19/dl/11_h7.pdf (accessed on 1 December 2020).

5. Barnes, C.M. Dental hygiene intervention to prevent nosocomial pneumonias. J. Evid. Based. Dent. Pract. 2014, 14, 103-114. [CrossRef] [PubMed]

6. Yoon, M.N.; Steele, C.M. Health care professionals' perspectives on oral care for long-term care residents: Nursing staff, speech-language pathologists and dental hygienists. Gerodontology 2012, 29, 525-535. [CrossRef] [PubMed]

7. Adachi, M.; Ishihara, K.; Abe, S.; Okuda, K. Professional oral health care by dental hygienists reduced respiratory infections in elderly persons requiring nursing care. Int. J. Dent. Hyg. 2007, 5, 69-74. [CrossRef] [PubMed]

8. Adachi, M.; Ishihara, K.; Abe, S.; Okuda, K.; Ishikawa, T. Effect of professional oral health care on the elderly living in nursing homes. Oral. Surg. Oral. Med. Oral. Pathol. Oral. Radiol. Endod. 2002, 94, 191-195. [CrossRef] [PubMed]

9. Abe, S.; Ishihara, K.; Adachi, M.; Sasaki, H.; Tanaka, K.; Okuda, K. Professional oral care reduces influenza infection in elderly. Arch. Gerontol. Geriatr. 2006, 43, 157-164. [CrossRef] [PubMed]

10. Report on Public Health Administration and Services, Ministry of Health, Labor and Welfare Japan. Available online: https: //www.mhlw.go.jp/toukei/saikin/hw/eisei/18/dl/kekka2.pdf (accessed on 1 December 2020).

11. Survey of Medical Institutions, Ministry of Health, Labor and Welfare Japan. Available online: https://www.mhlw.go.jp/toukei/ saikin/hw/iryosd/19/dl/02sisetu01.pdf (accessed on 1 December 2020).

12. Survey of the Working Environment of Japanese Dental Hygienists for the Fiscal Year 2019, Japan Dental Hygienists Association. Available online: https:/ / www.jdha.or.jp/pdf/aboutdh/r2-dh_hokoku.pdf (accessed on 1 December 2020).

13. Hopcraft, M.; McNally, C.; Ng, C.; Pek, L.; Pham, T.A.; Phoon, W.L.; Poursoltan, P.; Yu, W. Attitudes of the Victorian oral health workforce to the employment and scope of practice of dental hygienists. Aust. Dent. J. 2008, 53, 67-73. [CrossRef] [PubMed]

14. Hopcraft, M.; McNally, C.; Ng, C.; Pek, L.; Pham, T.A.; Phoon, W.L.; Poursoltan, P.; Yu, W. Working practices and job satisfaction of Victorian dental hygienists. Aust. Dent. J. 2008, 53, 61-66. [CrossRef] [PubMed]

15. Gibbons, D.E.; Corrigan, M.; Newton, J.T. A national survey of dental hygienists: Working patterns and job satisfaction. Br. Dent. J. 2001, 190, 207-210. [CrossRef]

16. Yavnai, N.; Bilder, L.; Sgan-Cohen, H.; Zini, A. Dental hygienists in Israel: Employment evaluation, job satisfaction, and training implications. J. Dent. Educ. 2012, 76, 1371-1376. [CrossRef] [PubMed]

17. Nomura, Y.; Okada, A.; Yamamoto, Y.; Kakuta, E.; Tomonari, H.; Hosoya, N.; Hanada, N.; Yoshida, N.; Takei, N. Factors Behind Leaving the Job and Rejoining it by the Japanese Dental Hygienist. Open Dent. J. 2020, 14, 355-361. [CrossRef]

18. Usui, Y.; Miura, H. Workforce re-entry for Japanese unemployed dental hygienists. Int. J. Dent. Hyg. 2015, 13, 74-78. [CrossRef] [PubMed]

19. Kanazawa, N. Prospects and Subjects of Dental Hygienists-Aiming at the Coordination with Medical Care and Elderly Care. Ann. Jpn. Prosthodont. Soc. 2014, 6, 267-272. [CrossRef] 